\title{
Galilean satellite ephemerides E5
}

\author{
J.H. Lieske \\ U.S.A. \\ e-mail: jay.lieske@jpl.nasa.gov \\ Received May 26; accepted September 30, 1997
}

Jet Propulsion Laboratory, California Institute of Technology, 4800 Oak Grove Dr., MS 301-150 Pasadena, 91109 California,

\begin{abstract}
New ephemerides of Jupiter's Galilean satellites are produced from an analysis of CCD astrometric data, Voyager-mission optical navigation images, mutual event observations, photographic plates, and eclipse timing observations. The resulting parameters, for use in the galsat computer software, are in the B1950 frame for use by the Galileo space mission. Results in the J2000 system are also available.
\end{abstract}

Key words: astrometry — celestial mechanics ephemerides — Planets and satellites: Jupiter

\section{Introduction}

This paper documents the Galilean satellite ephemerides designated as E5, which were delivered in support of the Galileo space mission to Jupiter. The E5 ephemerides supersede the E4 ephemerides, which were developed (Lieske 1994a) without using CCD astrometric data in order to assess the new data type. It is believed that the E5 ephemerides are better than the E3 and E4 ephemerides and they are recommended for general usage. The parameters of E5 are given in the B1950 system so that the galsat software (Lieske 1977) can be employed directly to compute coordinates in the B1950 frame, which has been adopted for the Galileo mission.

The ephemerides E2 (Lieske 1980) were developed prior to the Voyager mission and were based solely on an analysis of earth-based observations. The E2 ephemerides utilized mutual event data from 1973 (Aksnes \& Franklin 1976), photographic astrometric observations from 19671978 (Pascu 1977 1979), and Jovian satellite eclipse timings from 1878-1974 (Pickering 1907; Pierce 1974; Lieske 1980).

Post-Voyager mission ephemeris improvements yielded ephemerides E3, which included Voyager optical navigation astrometric data and Voyager-derived physical constants (Campbell \& Synnott 1985). The E3 ephemerides employed mutual event data from 1973 and 1979 (Aksnes et al. 1984), Voyager optical navigation astrometric measurements from 1979 (Synnott et al. 1982), additional photographic observations by D. Pascu from 1973-1979, and eclipse timings from 1652 to 1983 (Lieske 1986, 1987).

The initial pre-Galileo mission ephemerides were designated E4 (Lieske 1994a) and included extended mutual event data and photographic data, but no CCD observations, since they were still in the process of being evaluated. The E4 ephemerides employed the previously mentioned Voyager data, mutual event data from 1973 and 1979 corrected for phase effects by adding $\delta t$ to the observation time (Aksnes et al. 1986), photographic data and Jovian eclipse timings, as well as additional mutual event astrometric measurements from 1985 and 1991 (Aksnes et al. 1986; Franklin et al. 1991; Kaas et al. 1997; Descamps 1994; Goguen et al. 1988; Goguen 1994; Mallama 1992), and additional photographic observations from Pascu (1993) covering the interval 1980-1991. Threeyears' of CCD data from Flagstaff (Monet et al. 1994; Owen 1995) were evaluated, but not employed in developing the E4 ephemerides.

The E5 ephemerides represent the most current evolution of the Galilean satellite ephemerides and incorporate all of the above data types, including an evaluation the Doppler data of Ostro et al. (1992).

The 50 parameters which define the theory of motion of the Galilean satellites (Lieske 1977) could also be transformed in a manner such that the same galsat computer program can be employed to compute rectangular coordinates with their values being in the J2000 system. Documentation and an algorithm for such transformation of all galsat-related ephemerides (e.g., Lieske 1977, 1980; Arlot 1982; Vasundhara 1994) will be issued later. In the meantime the equatorial coordinates can be transformed in the following manner.

For the Galileo mission, all input quantities are in the B1950 frame and Earth equatorial coordinates 
transformation from B1950 to J2000 when necessary is done by the matrix multiplication

$r_{\mathrm{J} 2000}=A r_{\mathrm{B} 1950}$,

where the matrix $A$ could be taken from that recommended by IAU Commission 20 (West 1992),

$A=P_{\mathrm{IAU}} R_{3}(-0.525)$

with $P_{\text {IAU }}$ being the standard IAU precession matrix from B1950 to J2000 (Lieske 1979),

$P_{\mathrm{IAU}}=R_{3}\left(-z_{\mathrm{A}}\right) R_{2}\left(\theta_{\mathrm{A}}\right) R_{3}\left(-\zeta_{\mathrm{A}}\right)$

or $A$ could be taken from the earlier discussion of Standish (1982), which was developed for transforming from DE118 to DE200,

$A=R_{3}\left(+0^{\prime \prime} .00073\right) P_{\mathrm{IAU}} R_{3}\left(-0^{\prime \prime} .53160\right)$.

It essentially consists of a rotation $\Delta E$ in the B1950 equatorial plane from the FK4 origin to the dynamical equinox and then precessing from B1950 to J2000 using the IAU 1976 equatorial precession parameters $P_{\text {IAU }}$ (Lieske et al. 1977).

The matrix $A$ could also be derived from Lieske's discussion (1994b) on the precession of orbital elements,

$A=R_{1}\left(-\varepsilon_{\mathrm{J} 2000}\right) R_{3}\left(L^{\prime}\right) R_{1}\left(-J_{\mathrm{A}}\right) R_{3}(-L) R_{1}\left(\varepsilon_{\mathrm{B} 1950}\right)$.

For the Galileo mission, the method of Standish given in Eq. (4) is employed to precess from B1950 to J2000.

The rotation matrices $R_{i}$ are the standard matrices for rotations about the $x, y$, or $z$ axes for $i=1,2,3$ :

$$
\begin{aligned}
& R_{1}=\left[\begin{array}{ccc}
1 & 0 & 0 \\
0 & \cos \theta & \sin \theta \\
0 & -\sin \theta & \cos \theta
\end{array}\right] \\
& R_{2}=\left[\begin{array}{ccc}
\cos \theta & 0 & -\sin \theta \\
0 & 1 & 0 \\
\sin \theta & 0 & \cos \theta
\end{array}\right] \\
& R_{3}=\left[\begin{array}{ccc}
\cos \theta & \sin \theta & 0 \\
-\sin \theta & \cos \theta & 0 \\
0 & 0 & 1
\end{array}\right] .
\end{aligned}
$$

The various matrices mentioned in Eqs. (2), (4) and (5) are presented in Table 1 . The maximum difference in satellite coordinates, due to the different precessional transformations, is about $1.5 \mathrm{~km}$, so any of the previously mentioned matrices could be used in a practical situation.

\section{The basic parameters}

In the galsat-type ephemerides, the Jovicentric Earthequatorial coordinates of the Galilean satellites are computed as a function of 50 "galsat" parameters (Lieske
1977). The definitions of the basic parameters upon which the theory depends are given in Tables 2 and 3. It is seen that they are a combination of physical parameters and orbital elements.

In the E5 ephemerides, we employed the satellite masses $\left(\varepsilon_{1}-\varepsilon_{4}\right)$ and Jupiter pole which were determined by Campbell \& Synnott (1985) from their analysis of the Voyager data. The Jupiter pole is a function of the longitude of the origin of the coordinates $\psi$ [theory parameter $\left.\beta_{15}\right]$, and the inclination $I_{\mathrm{J}}$ of Jupiter's equator to Jupiter's orbit [theory parameter $\varepsilon_{25}$ ], with some dependence upon the Jupiter orbital inclination to the ecliptic [theory parameter $\varepsilon_{26}$ ], Jupiter's node $\Omega_{\mathrm{J}}$ [theory parameter $\beta_{22}$ ], and the obliquity $\varepsilon$ of the ecliptic [theory parameter $\varepsilon_{27}$ ]. The mass of the Jupiter system was that of JPL ephemeris DE140 (Standish \& Folkner 1995) Sun/Jupitersystem $=1047.3486$. Ephemerides E3 and E4 employed Jupiter system masses which are consistent with JPL ephemeris DE125 (Standish 1985), Sun/Jupiter-system $=1047.349$. The Jupiter pole employed was $\alpha_{\mathrm{J}}=268^{\circ} .001$ and $\delta_{\mathrm{J}}=64.504$ at the theory epoch JED 2443000.5 and in the B1950 frame. The rate of $\psi$ [theory parameter $\beta_{15}$ ] models the secular motion of Jupiter's pole from the theory epoch. Jupiter's oblateness parameters $J_{2}$ and $J_{4}$ were also taken from the Campbell \& Synnott analysis. They correspond to theory parameters $\varepsilon_{11}$ and $\varepsilon_{12}$ in Table 2 .

Over the years different tables of $\Delta T$ have been used for the calculation of Ephemeris Time (barycentric dynamical time TDB) minus Universal Time. The appropriate table of $\Delta T$ values depends upon what model of the Moon's tidal acceleration one adopts. The Earth's Moon was most often used to determine values of $\Delta T$ prior to 1955 because of its rapid motion. The derived values of $\Delta T$ effectively depend upon a partitioning into portions due to lunar tidal effects versus real changes in $\Delta T$. It essentially depends upon the parameter employed to describe the lunar tidal acceleration $\dot{n}_{\text {Moon }}$. The classical determination of $\dot{n}_{\text {Moon }}=-22.44 \mathrm{arcsec} / \mathrm{cy}^{2}$ by Spencer Jones (1939) was employed for the E1 and E2 (Lieske 1980) ephemerides by means of the Brouwer (1952) and Martin (1969) values of $\Delta T$, which were on the Spencer Jones system.

The Morrison and Ward (1975) value of $\dot{n}_{\text {Moon }}=$ $-26.0 \mathrm{arcsec} / \mathrm{cy}^{2}$ was used for E3, E4 and E5. Tables of $\Delta T$ given by Stephenson \& Morrison (1984) can be adjusted for any $\dot{n}_{\text {Moon }}$ by the technique noted in Lieske (1987) for times prior to 1955.5 by computing

$$
\Delta T\left(\dot{n}_{\text {Moon }}\right)=\Delta T_{\text {Morrison }}-0.911\left(\dot{n}_{\text {Moon }}+26\right) T_{0}^{2} \text { sec }
$$

where $T_{0}$ is measured in centuries from the 1955.5 epoch of Morrison (1980). The theory parameters of E1 and E2 are consistent with the Spencer-Jones value of $\dot{n}_{\text {Moon }}$, while those for E3 through E5 are consistent with that of Morrison and Ward. 
Table 1. Matrices for precession from B1950 to J2000

$$
\begin{array}{lrr}
\text { Eq. (2): Commission } 20 \text { matrix from } P_{\mathrm{IAU}} R_{3}(-0.525) \\
& & \\
0.9999256794956877 & -0.0111814832204662 & -0.0004859003815359 \\
0.0111814832391717 & 0.9999374848933135 & -0.0000271625947142 \\
0.0048590037723143 & -0.0000271702937440 & 0.9999881946023742
\end{array}
$$

Eq. (4): Standish matrix from $R_{3}\left(+0^{\prime \prime} .00073\right) P_{\mathrm{IAU}} R_{3}\left(-0^{\prime \prime} .53160\right)$

$\begin{array}{lrr}0.9999256791774783 & -0.0111815116768724 & -0.0048590038154553 \\ 0.0111815116959975 & 0.9999374845751042 & -0.0000271625775175 \\ 0.0048590037714450 & -0.0000271704492210 & 0.9999881946023742\end{array}$

Eq. (5): Lieske matrix from $R_{1}\left(-\varepsilon_{\mathrm{J} 2000}\right) R_{3}\left(L^{\prime}\right) R_{1}\left(-J_{\mathrm{A}}\right) R_{3}(-L) R_{1}\left(\varepsilon_{\mathrm{B} 1950}\right)$

$\begin{array}{lrr}0.9999256795268940 & -0.0111810778339439 & -0.0004859930159015 \\ 0.0111810775053504 & 0.9999374894281627 & -0.0000272382503387 \\ 0.0048599309149990 & -0.0000271030297995 & 0.9999881900987267\end{array}$

\begin{tabular}{|c|c|c|c|}
\hline \multicolumn{2}{|c|}{ Epsilon Parameter } & Generating value & Description \\
\hline 1 & $m_{1}$ & $449.710^{-7}\left(1+\epsilon_{1}\right)$ & Mass of Satellite I relative to Jupiter \\
\hline 2 & $m_{2}$ & $252.910^{-7}\left(1+\epsilon_{2}\right)$ & Mass of Satellite II relative to Jupiter \\
\hline 3 & $m_{3}$ & $798.810^{-7}\left(1+\epsilon_{3}\right)$ & Mass of Satellite III relative to Jupiter \\
\hline 4 & $m_{4}$ & $450.410^{-7}\left(1+\epsilon_{4}\right)$ & Mass of Satellite IV relative to Jupiter \\
\hline 5 & $S / J$ & $1047.355\left(1+\epsilon_{5}\right)$ & Mass of Sun relative to Jupiter \\
\hline 6 & $n_{1}$ & $203.488954208\left(1+\epsilon_{6}\right)$ & Mean motion of Satellite I, deg/day \\
\hline 7 & $n_{2}$ & $101.374723445\left(1+\epsilon_{7}\right)$ & Mean motion of Satellite II, deg/day \\
\hline 8 & $n_{4}$ & $21.571071403\left(1+\epsilon_{8}\right)$ & Mean motion of Satellite IV, deg/day \\
\hline 9 & $\lambda_{\mathrm{A}}$ & $180^{\circ} \epsilon_{9} / \pi$ & Amplitude of free libration, $\lambda_{\mathrm{A}}$ in $\mathrm{deg}, \epsilon_{9}$ in $\mathrm{rad}$ \\
\hline 10 & $n_{\mathrm{J}}$ & $8.309121571210^{-2}\left(1+\epsilon_{10}\right)$ & Mean motion of Jupiter, deg/day \\
\hline 11 & $J_{2}$ & $0.0148485\left(1+\epsilon_{11}\right)$ & Jupiter $J_{2}$ \\
\hline 12 & $J_{4}$ & $-8.10710^{-4}\left(1+\epsilon_{12}\right)$ & Jupiter $J_{4}$ \\
\hline 13 & $R_{\mathrm{J}}$ & $71420\left(1+\epsilon_{13}\right)$ & Radius of Jupiter, km \\
\hline 14 & $P_{\mathrm{J}}$ & $9.924825\left(1+\epsilon_{14}\right)$ & Period of Jupiter rotation, hr \\
\hline 15 & $3(C-A) / 2 C$ & $0.111\left(1+\epsilon_{15}\right)$ & Ratio of Jupiter moments of inertia \\
\hline 16 & $e_{11}$ & $46510^{-7}\left(1+\epsilon_{16}\right)$ & Primary eccentricity of Satellite I, rad \\
\hline 17 & $e_{22}$ & $82510^{-7}\left(1+\epsilon_{17}\right)$ & Primary eccentricity of Satellite II, rad \\
\hline 18 & $e_{33}$ & $1516410^{-7}\left(1+\epsilon_{18}\right)$ & Primary eccentricity of Satellite III, rad \\
\hline 19 & $e_{44}$ & $7372510^{-7}\left(1+\epsilon_{19}\right)$ & Primary eccentricity of Satellite IV, rad \\
\hline 20 & $e_{\mathrm{J}}$ & $0.0484602472\left(1+\epsilon_{20}\right)$ & Eccentricity of Jupiter \\
\hline 21 & $c_{11}$ & $475610^{-7}\left(1+\epsilon_{21}\right)$ & Primary sine inclination of Satellite I \\
\hline 22 & $c_{22}$ & $8149010^{-7}\left(1+\epsilon_{22}\right)$ & Primary sine inclination of Satellite II \\
\hline 23 & $c_{33}$ & $3110810^{-7}\left(1+\epsilon_{23}\right)$ & Primary sine inclination of Satellite III \\
\hline 24 & $c_{44}$ & $4746010^{-7}\left(1+\epsilon_{24}\right)$ & Primary sine inclination of Satellite IV \\
\hline 25 & $I_{\mathrm{J}}$ & $3.10401\left(1+\epsilon_{25}\right)$ & Inclination of Jupiter orbit to Jupiter equator, deg \\
\hline 26 & $J$ & $1.30691\left(1+\epsilon_{26}\right)$ & Inclination of Jupiter orbit to ecliptic, deg \\
\hline 27 & $\epsilon$ & $23^{\circ} 26^{\prime} 44^{\prime \prime} .84\left(1+\epsilon_{27}\right)$ & Inclination (Obliquity) of ecliptic to Earth equator deg \\
\hline 28 & $n_{\mathrm{S}}$ & $3.345973389610^{-2}\left(1+\epsilon_{28}\right)$ & Mean motion of Saturn, deg/day \\
\hline
\end{tabular}

Table 2. Definition of theory parameters $\varepsilon$ 
Table 3. Definition of theory parameters $\beta$

\begin{tabular}{|c|c|c|c|}
\hline \multicolumn{2}{|c|}{ Beta Parameter } & Epoch value (deg) & Description \\
\hline 1 & $\ell_{1}$ & $106^{\circ} .03042+\beta_{1}$ & Mean longitude of Satellite I \\
\hline 2 & $\ell_{2}$ & $175.74748+\beta_{2}$ & Mean longitude of Satellite II \\
\hline 3 & $\ell_{3}$ & {$\left[120.60601-\frac{1}{2} \beta_{1}+\frac{3}{2} \beta_{2}\right]$} & Mean longitude of Satellite III \\
\hline 4 & $\ell_{4}$ & $84.51861+\beta_{4}$ & Mean longitude of Satellite IV \\
\hline 5 & $\phi_{\lambda}$ & $\beta_{5}$ & Free Libration $\begin{aligned} \psi_{1}-3 \psi_{2}+2 \psi_{3} & =\pi+\epsilon_{9} \sin \phi_{\lambda} \\
& =180^{\circ}+\lambda_{\mathrm{A}} \sin \phi_{\lambda}\end{aligned}$ \\
\hline 6 & $\pi_{1}$ & $4.51172+\beta_{6}$ & Proper periapse of Satellite I \\
\hline 7 & $\pi_{2}$ & $74.53051+\beta_{7}$ & Proper periapse of Satellite II \\
\hline 8 & $\pi_{3}$ & $174.85831+\beta_{8}$ & Proper periapse of Satellite III \\
\hline 9 & $\pi_{4}$ & $336^{\circ} 02667+\beta_{9}$ & Proper periapse of Satellite IV \\
\hline 10 & $\Pi_{\mathrm{J}}$ & $13.30364+\beta_{10}$ & Longitude of perihelion of Jupiter \\
\hline 11 & $\omega_{1}$ & $242.73706+\beta_{11}$ & Proper node of Satellite I \\
\hline 12 & $\omega_{2}$ & $95^{\circ} 28556+\beta_{12}$ & Proper node of Satellite II \\
\hline 13 & $\omega_{3}$ & $125^{\circ} .14673+\beta_{13}$ & Proper node of Satellite III \\
\hline 14 & $\omega_{4}$ & $317.89250+\beta_{14}$ & Proper node of Satellite IV \\
\hline 15 & $\psi$ & $316.73369+\beta_{15}$ & Longitude of origin of coordinates (Jupiter's pole) \\
\hline 16 & $G^{\prime}$ & $31.9785280244+\beta_{16}$ & Mean anomaly of Saturn \\
\hline 17 & $G$ & $30^{\circ} 3784120168+\beta_{17}+\delta G$ & Mean anomaly of Jupiter \\
\hline 18 & $\phi_{1}$ & $172.84\left(1-0.014 \epsilon_{20}\right)+\beta_{18}$ & Phase angle in solar $(A / R)^{3}$ with angle $2 G^{\prime}-G$ \\
\hline 19 & $\phi_{2}$ & $47^{\circ} .03\left(1-0.156 \epsilon_{20}\right)+\beta_{19}$ & Phase angle in solar $(A / R)^{3}$ with angle $5 G^{\prime}-2 G$ \\
\hline 20 & $\phi_{3}$ & $259.18+\beta_{20}$ & Phase angle in solar $(A / R)^{3}$ with angle $G^{\prime}-G$ \\
\hline 21 & $\phi_{4}$ & $157^{\circ} .12\left(1+0.0014 \epsilon_{20}\right)+\beta_{21}$ & Phase angle in solar $(A / R)^{3}$ with angle $2 G^{\prime}-2 G$ \\
\hline 22 & $\Omega_{\mathrm{J}}$ & $99.95326+\beta_{22}$ & Longitude ascending node of Jupiter's orbit on ecliptic \\
\hline \multicolumn{2}{|c|}{ Beta Symbol } & Rate (deg/day) & Description \\
\hline 1 & $\dot{\ell}_{1}$ & $203.488954208\left(1+\epsilon_{6}\right)$ & Mean motion of Satellite I \\
\hline 2 & $\dot{\ell}_{2}$ & $101: 374723445\left(1+\epsilon_{7}\right)$ & Mean motion of Satellite II \\
\hline 3 & $\dot{\ell}_{3}$ & $\begin{array}{l}{\left[50.3176080635\left\{1-2 \epsilon_{6}+3 \epsilon_{7}\right.\right.} \\
\left.\left.-0.02204518497\left(\epsilon_{6}-\epsilon_{7}\right)\right\}\right]\end{array}$ & ${ }_{7}$ Mean motion of Satellite III \\
\hline 4 & $\dot{\ell}_{4}$ & $21.571071403\left(1+\epsilon_{8}\right)$ & Mean motion of Satellite IV \\
\hline 5 & $\dot{\phi}_{\lambda}$ & $\sqrt{L}(=0.17379190+\cdots)$ & Rate of free libration (Fiche Table A.30) \\
\hline 6 & $\dot{\pi}_{1}$ & $(0 \circ 16138586+\cdots)$ & Proper periapse rate of Satellite I \\
\hline 7 & $\dot{\pi}_{2}$ & $(0.04726307+\cdots)$ & Proper periapse rate of Satellite II \\
\hline 8 & $\dot{\pi}_{3}$ & $(0.00712734+\cdots)$ & Proper periapse rate of Satellite III \\
\hline 9 & $\dot{\pi}_{4}$ & $(0.00184000+\cdots)$ & Proper periapse rate of Satellite IV \\
\hline 10 & $\dot{\Pi}_{\mathrm{J}}$ & 0 & \\
\hline 11 & $\dot{\omega}_{1}$ & $\left(-0^{\circ} 13279386+\cdots\right)$ & Proper node rate of Satellite I \\
\hline 12 & $\dot{\omega}_{2}$ & $\left(-0^{\circ} .03263064+\cdots\right)$ & Proper node rate of Satellite II \\
\hline 13 & $\dot{\omega}_{3}$ & $(-0.00717703+\cdots)$ & Proper node rate of Satellite III \\
\hline 14 & $\dot{\omega}_{4}$ & $(-0.00175934+\cdots)$ & Proper node rate of Satellite IV \\
\hline 15 & $\dot{\psi}$ & $(-0.00000208+\cdots)$ & Longitude of origin rate \\
\hline 16 & $\dot{G}^{\prime}$ & $3.3459733896 \cdot 10^{-2}\left(1+\epsilon_{28}\right)$ & Mean motion of Saturn \\
\hline 17 & $\dot{G}$ & $8.3091215712 \cdot 10^{-2}\left(1+\epsilon_{10}\right)$ & Mean motion of Jupiter \\
\hline 18. & & 0 & \\
\hline
\end{tabular}




\section{The observations}

A variety of different observational data types were employed in developing ephemerides E5. A new and very powerful data type of CCD observations from the U.S. Naval Observatory Flagstaff Station was used for the first time, together with very accurate Voyager optical navigation data from 1979 and the mutual event observations 1973-1991, photographic observations of D. Pascu from 1967-1993 and Jovian eclipse timings from 16521983. Doppler observations from 1987-1991 were employed to assess the value of the Doppler data and evaluate the ephemerides.

Table 4. Observational data employed for ephemeris E5

\begin{tabular}{lllr} 
Data span & observable type & observ. & \% chg \\
\hline $1992-1994$ & CCD data, Flagstaff ra \& dec & 870 & -52.6 \\
1979 & Voyager opnav ra \& dec & 366 & -19.0 \\
$1973-1991$ & mutual events ra \& dec & 860 & -55.5 \\
$1967-1993$ & photographic ra \& dec & 8462 & -3.2 \\
$1652-1983$ & eclipse timings & 15711 & +2.7 \\
1994 & CCD data, Table Mountain & 72 & +68.3 \\
$1987-1991$ & Doppler & 50 & -55.6 \\
\hline
\end{tabular}

By intercomparing various data types one learns of the strengths and weaknesses of each individual type of data and discovers inconsistencies among the data types. The data are described in Table 4, which also gives the percentage change in weighted sum-of-squares for ephemeris E5 relative to ephemeris E3. A plus sign indicates an increase and a minus sign indicates a decrease in the weighted residuals. The various data types were combined by weighting each observation by the reciprocal of its squared a priori standard deviation. A common data set (including weights) was employed to evaluate all ephemerides so that one can compare the relative merits of a given ephemeris to a common data set. Thus, although no CCD observations were employed in the development of ephemeris E3, the residuals of the CCD data employed in this paper are also given for ephemeris E3 so that the reader can make meaningful comparisons.

In order to more closely compare the various ephemerides with the different data types, we present in Table 5 the residuals of unit weight for each data type for the different ephemerides E2 through E5 by Lieske, as well as for the Bureau des Longitudes' ephemeris G5 (Arlot 1982). In comparing Table 4 with Table 5 it should be remembered that Table 4 is related to the square of the residuals while Table 5 employs the square root of the sum-of-squares. The comparison for Flagstaff CCD data, for example, for Table 5 would indicate that the Table 4 entry should be about $(29 / 43)^{2}-1=-54.5 \%$ for the E5 vs E3 comparison.
Table 5. Observational rms residuals for various ephemerides

\begin{tabular}{lrrrrr}
\hline Observable type & E2 & G5 & E3 & E4 & E5 \\
\hline CCD Flagstaff, mas & 43 & 40 & 43 & 32 & 29 \\
Voyager opnav, mas & 1309 & 1334 & 929 & 904 & 820 \\
mutual events, mas & 62 & 53 & 62 & 47 & 46 \\
photographic, mas & 107 & 106 & 106 & 104 & 104 \\
eclipse timings, sec & 55.5 & 74.5 & 53.2 & 53.9 & 53.9 \\
CCD Table Mtn, mas & 43 & 73 & 41 & 52 & 53 \\
Doppler, Hz & 15.3 & 18.4 & 13.7 & 11.7 & 11.9 \\
\hline
\end{tabular}

\section{1. $C C D$ observations}

The new CCD observations were made at the U.S. Naval Observatory Flagstaff Station (A. Monet et al. 1994) during the years 1993-1995, employing techniques developed by D. Monet and described in Monet et al. (1992) and in Monet \& Monet (1992). The Flagstaff data were processed at JPL by W. Owen who produced normal-point residuals, typically from 30-50 CCD "exposures", for the author using ephemeris E3. Those residuals were then employed by the author to generate pseudo-observable "normal-point observations" by adding the residual to an artificiallyconstructed computed position at the mean time of the CCD exposures using the same ephemeris which was employed in computing the CCD residuals. Such a "normal point observation" could be employed with other astrometric data in an analysis of the observations, and should represent a valid description of the actual CCD observations. Additionally, the pseudo-observations will serve the purpose of archiving the CCD observations in convenient form. In processing the CCD data Owen would estimate the pointing and orientation parameters and employ a single telescope scale factor (modified for refraction and atmospheric effects) for all the Flagstaff data and he would use a single ephemeris (viz. E3) which was not adjusted in the reduction process. If that procedure is valid, then the pseudo-observables generated should behave like valid observational data, viz. the residuals should decrease if one employs a better ephemeris with the original pseudoobservables. It was for this reason that ephemeris E3 was intentionally employed - it was known to need some correction and we desired to explore the validity of the process of constructing normal point pseudo-observables. If the normal points were constructed instead on a different ephemeris, then the pseudo-observables differed by less than $15 \mathrm{~km}(0$ ".005) from those generated via ephemeris E3, even though the residuals might actually be significantly different using the two ephemerides. That $15-\mathrm{km}$ reproducibility of the normal points is a good indication of the intrinsic accuracy of the CCD data.

Some less-accurate CCD data from the JPL Table Mountain Facility (Owen 1995) were also employed, although with hindsight they probably should not have been 

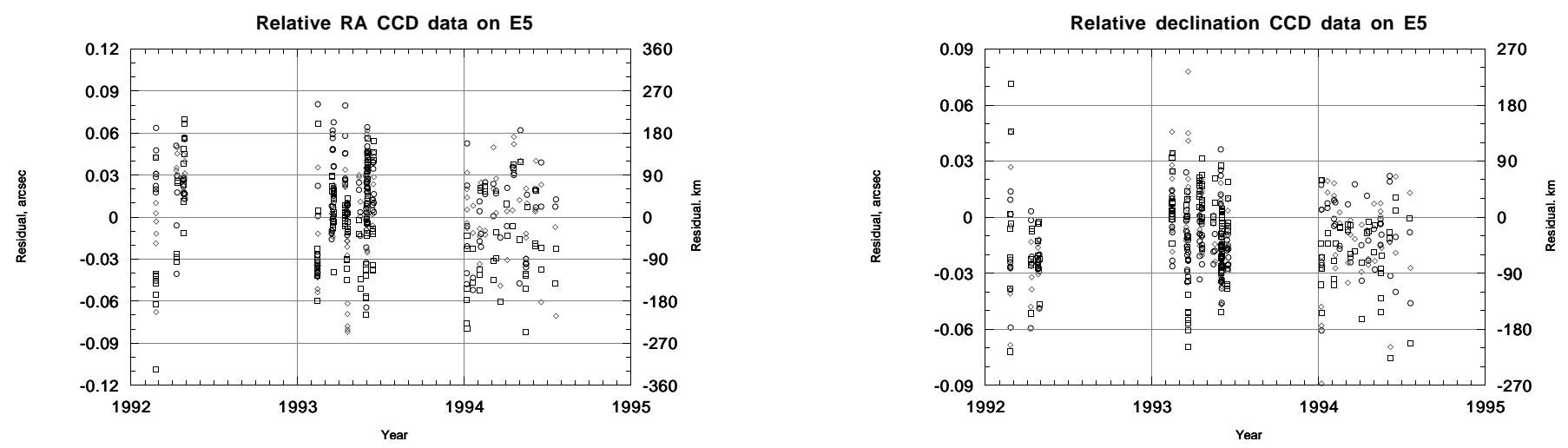

Fig. 1. Residuals in right ascension (left) and declination (right) for Flagstaff CCD observations relative to Satellite 1 using ephemeris E5. The observations of Europa relative to Io are indicated by a $\circ$, those of Ganymede by a $\square$, and those of Callisto by a $\diamond$

included in developing E5. They did not exhibit the reduction of residuals with a better ephemeris, and that is believed to be due to the fact that there were too few Table Mountain data to adequately separate the orbital effects from the telescope effects.

The CCD data were processed using Lambert scattering to compute the offset between the center of light and center of figure (Lindegren 1977) and it is believed that the dominant remaining unmodeled error source in these data is due to albedo variations across the disk of the satellites. Recent estimates of the albedo variations by several scientists (Goguen 1994; Mallama 1993; Riedel 1994; Gaskell 1995) are not entirely consistent and for the Galileo-mission ephemerides it was decided to limit the processing to computation of the difference between center of light and center of figure due to Lambert scattering only, since it represents a reasonable first approximation to the scattering properties of the satellites if one excludes albedo variations (viz., effects which depend upon features on the satellites and which vary with planetocentric longitude of the central disc). The extrapolation of Voyager-derived scattering properties (which occurred at high phase angle) to the scattering properties of the satellites at low phase angle as observed from the Earth is not entirely satisfactory and the several efforts done to date are not entirely consistent with one another. It is hoped that some series of observations made from the Hubble Space Telescope will resolve the problems. Employment of Lambert scattering is a useful first-approximation. The differences between Lambert, Minnaert or Hapke scattering laws is minor compared to the albedo variations introduced by physical features on the satellites, which may introduce center-of-light relative to center-of-figure variations on the order of $75-100 \mathrm{~km}$.

The Flagstaff CCD data were weighted using a standard deviation of 0 .' 03 , which corresponds to about $90 \mathrm{~km}$ for these earth-based observations. The Table Mountain data were weighted using a standard deviation of 0 ".05, corresponding to about $150 \mathrm{~km}$.

\subsection{Voyager optical navigation data}

During the Voyager mission in 1979, some optical navigation images of the Jovian satellites were taken from the spacecraft for use in navigating the spacecraft to the Jovian encounter. We have 183 observations of the Jovian satellites in right ascension and in declination, made during the Voyager I and Voyager II encounters (Synnott et al. 1982). The optical navigation images are analogous to earth-based astrometric observations of the satellites except that the "opnav" images are taken by an "observer" much closer to the Jovian system (typically $13-95$ light seconds from the satellites). At 5 $10^{6} \mathrm{~km}$ from Jupiter, one arcsec corresponds approximately to $25 \mathrm{~km}$. Additionally, the spacecraft-based observations are the result of analyzing extended satellite images. By inferring the center of the satellite from observations of the limb, the Voyager data do not have the center-of-light vs center-of-figure problems which are common to disk-integrated images such as those contained in CCD observations and photographic plates and mutual events. The Voyager data were weighted using a standard deviation of $1{ }^{\prime \prime} .0$ (as seen at the spacecraft's distance from Jupiter). For spacecraft-to-satellite distances of 13 - 95 light seconds, the $1^{\prime \prime} .0$ corresponds to 19 and 140 $\mathrm{km}$ respectively for these spacecraft-based observations. The Voyager optical navigation residuals on ephemeris E5 are depicted for right ascension and declination in Fig. 2.

\subsection{Mutual event astrometric data}

Since 1973 there have been successful campaigns to observe the mutual event seasons every six years, when the Jovian satellites eclipse and occult one another as the Sun and the Earth pass through the plane of the Jovian equator, in which the satellite orbits lie. Aksnes and colleagues 

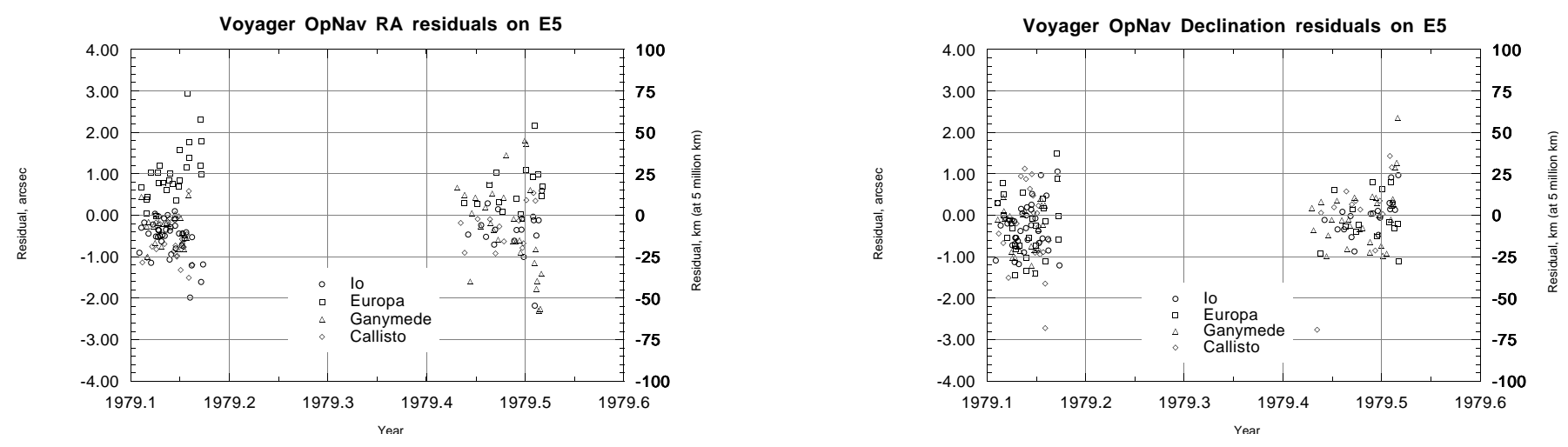

Fig. 2. Residuals in right ascension (left) and declination (right) for the Voyager optical navigation observations using ephemeris E5. The ordinate is in arcsec with an approximate corresponding linear distance scale on the right. Jupiter-relative observations of Io are indicated by $\circ$, Europa by $\square$, Ganymede by $\triangle$, and Callisto by $\diamond$
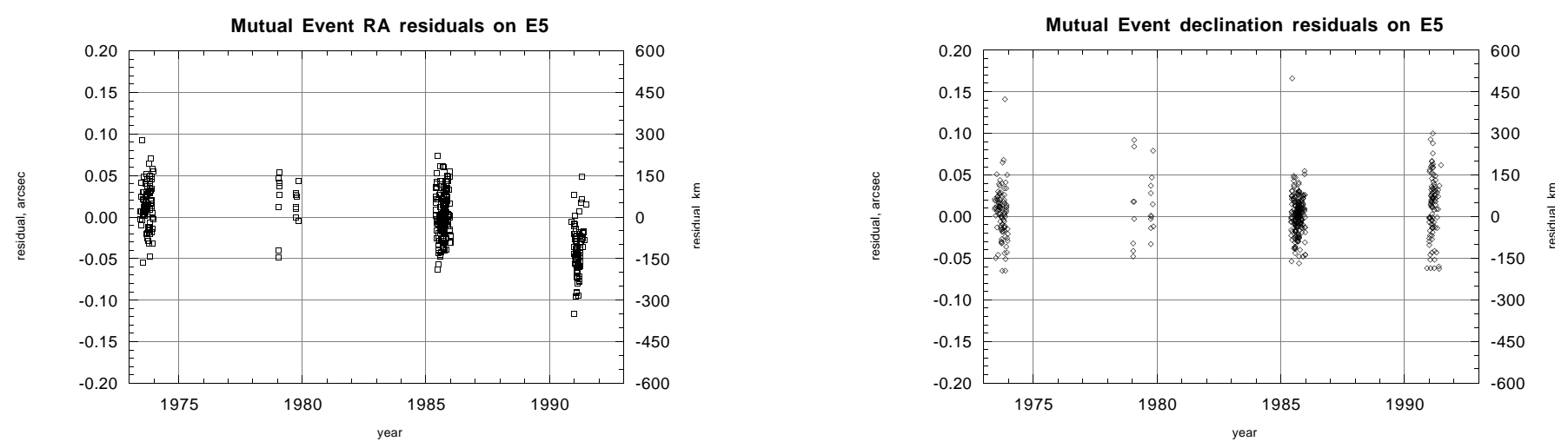

Fig. 3. Residuals in right ascension (left) and declination (right) for astrometric mutual event observations using ephemeris E5. The ordinate is in arcsec with an approximate corresponding linear distance scale on the right

(Aksnes 1974, 1984; Aksnes \& Franklin 1978, 1990), along with Arlot and colleagues (Arlot 1978, 1984, 1990, 1996), have made predictions of such mutual events available to scientists throughout the world and have organized scientific programs to observe the mutual events. Aksnes' team has produced astrometric separations of the satellites, at times near the mid-event times, which are very useful for ephemeris development purposes.

The early Galilean satellite ephemerides E1 and E2 (Lieske 1980) employed the Aksnes data from 1973 (Aksnes \& Franklin 1976) and 1979 (Aksnes et al. 1984) and were affected by the phase offsets between eclipses and occultations which led Aksnes et al. (1986) to recommend that $\delta t$ be added to the published observation times for the 1973 and 1979 data. The ephemerides E3 were generated using the recommended additions of $\delta t$ to the observation times in processing the 1973 and 1979 mutual events astrometric data.

In the processing of mutual event observations by the Aksnes team in 1985 (Franklin et al. 1991) and 1991 (Kaas et al. 1997), it was intended that no value of $\delta t$ would be required but that instead the authors would incorporate the phase effects into their published times and separa- tions. However, the effects were added in the incorrect direction for the published data and hence it is recommended (Aksnes 1993; Franklin 1993; Lieske 1995) that the 1985 and 1991 Aksnes data be employed by adding twice the published values of the $\delta t$ phase corrections to the observation times. Essentially the first addition of $\delta t$ removes the erroneous application of the phase effects with the incorrect sign and the second application of $\delta t$ actually corrects for the phase problem. Additionally, some infra-red astrometric mutual event separations were obtained from Goguen et al. (1988) in 1985 as well as in 1991 (Goguen 1994). Astrometric separations from the 1991 mutual event season which were employed in the development of E5 were also published by Mallama (1992a), Spencer (1993) and by Descamps (1994).

The mutual event data were weighted using standard deviations of $0^{\prime \prime} .020$ to $0^{\prime \prime} .045$, which corresponds to $60 \mathrm{~km}$ and 140 respectively for these earth-based observations. The typical weight corresponds to a standard deviation of $0^{\prime \prime} .030$ or $90 \mathrm{~km}$.

The obvious offset in right ascension residuals for the 1991 mutual event season depicted in Fig. 3 is believed not to be due to ephemeris errors, but rather is due to albedo 

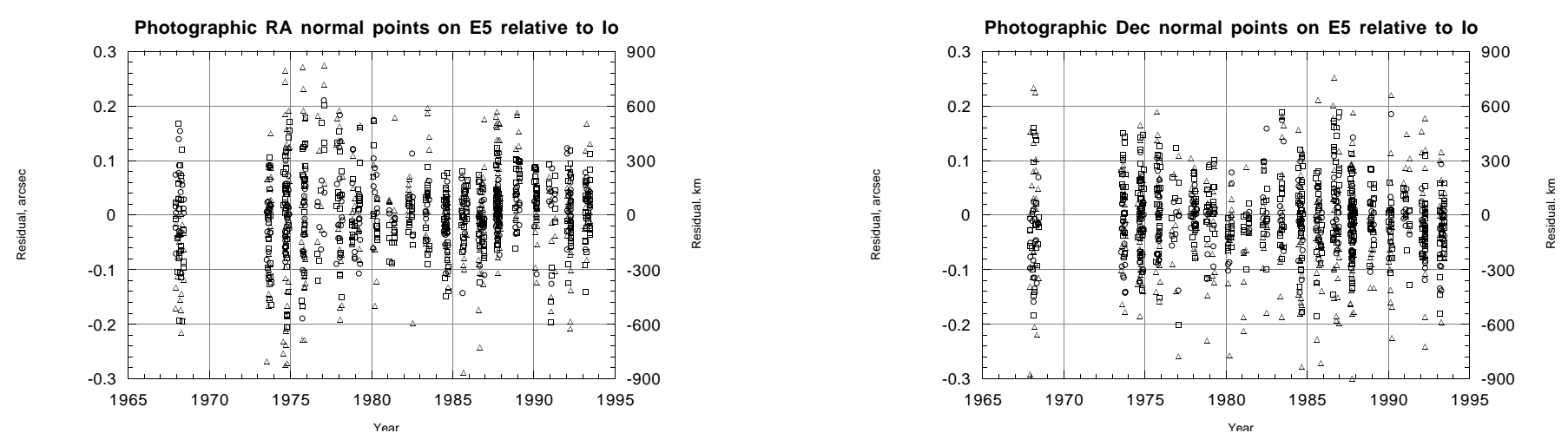

Fig. 4. Residuals in right ascension (left) and declination (right) for photographic observations relative to Io using ephemeris E5. The residuals for exposures of a given satellite on each plate have been combined to produce a normal point for each plate. Observations of Europa relative to Io are indicated by a $\circ$, those of Ganymede by a $\square$ and those of Callisto by a $\diamond$

effects since almost all of the 1991 mutual event observations involved Io and were made at comparable longitudes on the satellite disk. The CCD and photographic data, for example, show no such offset and those data were sampled at various longitudes.

\subsection{Photographic observations}

The long and valuable series of photographic observations made by D. Pascu of the U.S. Naval Observatory have been an essential ingredient of the Galilean satellite ephemerides since the first development of the Galsat software. In an extended series of observations 1967-1993, Pascu (1977, 1979, 1993, 1994) provided astrometric observations of the satellites. He pioneered the development of neutral density filters to enable the accurate observation of the Galilean satellites on a regular basis. The Pascu data were reduced using a single scale factor (modified by adjustments for refraction for each observation) for the ensemble of observations, as determined by Pascu. Additionally, a correction to the Pascu scale was applied for a refraction-related effect, amounting to a relative change in scale of $-58^{\prime \prime} \cdot 2 / 206265$, which probably resulted from the manner in which the plate scale was originally determined.

The photographic data from 1967 through 1975 were weighted using a standard deviation of 0 ". 13 per exposure, while those from 1976 onwards were weighted using a standard deviation of 0 ".09 per exposure, corresponding to position uncertainties of $400 \mathrm{~km}$ and $275 \mathrm{~km}$, respectively, for each exposure. A photographic plate typically consisted of 4 exposures of each satellite.

The residuals on E5 for photographic observations are plotted in Fig. 4. In the figure, normal-point residuals are presented for each photographic plate, in order to make the comparison with the normal-point CCD observations more feasible. In the plots, the residuals for all exposures of a given satellite on a single plate are averaged into a single normal-point residual.

\subsection{Jupiter eclipse timings}

The Jovian eclipse timings, representing the classical observations of the Galilean satellites back to the 17th century, were discussed in Lieske (1986a,b). The early data are from the Pingré 17th century collection later published by Bigourdan (1901), and from the Delisle collection (Bigourdan 1897). The book on 17th century astronomy by Pingré published by Bigourdan was originally scheduled for publication 100 years earlier by Pingré. But Pingré's death and the French revolution intervened, and the printer's proof copies were destroyed as scrap paper. It was only 100 years later that a copy of the proofs was found and ultimately published by the Paris Academy. The manuscript collection of J.-N. Delisle contains a wealth of historically and scientifically interesting observations of Galilean satellite eclipses. These two collections effectively re-construct the "lost" Delambre collection.

We employed satellite radii of $1815,1569,2631$ and $2400 \mathrm{~km}$ for Io through Callisto, respectively (Davies et al. 1985), in reducing the eclipse timings.

Additionally, the series of eclipse observations by Pickering from 1878-1903 (Pickering 1907) and those accumulated by Pierce (1974), together with those of many amateur astronomers, especially those coordinated by B. Loader and J. Westfall, were employed. Finally, a few eclipse timings by Mallama (1992b) taken in 1990-91 were analyzed.

The eclipse timing data were employed with average standard deviations between $44 \mathrm{~s}$ for Io and $150 \mathrm{~s}$ for Callisto with a mean of $63 \mathrm{~s}$, which correspond to position uncertainties of $775 \mathrm{~km}$ for Io, $1225 \mathrm{~km}$ for Callisto, and $800 \mathrm{~km}$ on the average for all satellites. The residuals appear visually similar to those depicted in Lieske (1986a) and therefore they are not presented here again. 
Table 6. Values of theory parameters $\varepsilon$ and $\beta$ for E5 in B1950 frame [see also Tables 2 and 3]

\begin{tabular}{|c|c|c|c|c|c|}
\hline$\overline{\text { Parameter }}$ & Related to & Value & Parameter & Related to & $\overline{\text { Value }}$ \\
\hline$\varepsilon_{1}$ & $m_{1}$ & $0.046323( \pm 0.000813)$ & $\varepsilon_{26}$ & $J$ & $-0.000137( \pm 0.000117)$ \\
\hline$\varepsilon_{2}$ & $m_{2}$ & $-0.000906( \pm 0.001394)$ & $\varepsilon_{27}$ & $\epsilon$ & $0.000000( \pm 0.000004)$ \\
\hline$\varepsilon_{3}$ & $m_{3}$ & $-0.022997( \pm 0.000276)$ & $\varepsilon_{28}$ & $n_{S}$ & $0.000000( \pm 0.000001)$ \\
\hline$\varepsilon_{4}$ & $m_{4}$ & $0.258508( \pm 0.000537)$ & $\beta_{1}$ & $\ell_{1}$ & $0.046767( \pm 0.00218)$ \\
\hline$\varepsilon_{5}$ & $S / J$ & $2009.3457 E-07( \pm 8.12 E-07)$ & $\beta_{2}$ & $\ell_{2}$ & $-0.015865( \pm 0.000835)$ \\
\hline$\varepsilon_{6}$ & $n_{1}$ & $7.7760 E-09( \pm 0.549 E-09)$ & $\beta_{3}$ & $\ell_{3}$ & {$\left[=-\frac{1}{2} \beta_{1}+\frac{3}{2} \beta_{2}\right]$} \\
\hline$\varepsilon_{7}$ & $n_{2}$ & $12.7230 E-09( \pm 1.04 E-09)$ & $\beta_{4}$ & $\ell_{4}$ & $-0.074023( \pm 0.001950)$ \\
\hline$\varepsilon_{8}$ & $n_{4}$ & $-10.4916 E-09( \pm 4.90 E-09)$ & $\beta_{5}$ & $\phi_{\lambda}$ & $199.676608( \pm 1.57)$ \\
\hline$\varepsilon_{9}$ & $\lambda_{\mathrm{A}}$ & $11.2104 E-04( \pm 0.391 E-04) \mathrm{rad}$ & $\beta_{6}$ & $\pi_{1}$ & $92.576366( \pm 19.9)$ \\
\hline$\varepsilon_{10}$ & $n_{\mathrm{J}}$ & $1.63 E-05( \pm 0.13 E-05)$ & $\beta_{7}$ & $\pi_{2}$ & $80.335825( \pm 1.35)$ \\
\hline$\varepsilon_{11}$ & $J_{2}$ & $-0.007576( \pm 0.000066)$ & $\beta_{8}$ & $\pi_{3}$ & $13.325727( \pm 0.150)$ \\
\hline$\varepsilon_{12}$ & $J_{4}$ & $-0.275934( \pm 0.00631)$ & $\beta_{9}$ & $\pi_{4}$ & $-0.739863( \pm 0.0152)$ \\
\hline$\varepsilon_{13}$ & $R_{\mathrm{J}}$ & $-0.000308( \pm 0.000057)$ & $\beta_{10}$ & $\Pi_{J}$ & $0.166302( \pm 0.00344)$ \\
\hline$\varepsilon_{14}$ & $P_{\mathrm{J}}$ & $9.5 E-06( \pm 102 . E-06)$ & $\beta_{11}$ & $\omega_{1}$ & $69.597506( \pm 0.788000)$ \\
\hline$\varepsilon_{15}$ & $3(C-A) / 2 C$ & $-0.170000( \pm 0.0676)$ & $\beta_{12}$ & $\omega_{2}$ & $5.155556( \pm 0.0495)$ \\
\hline$\varepsilon_{16}$ & $e_{11}$ & $-0.995346( \pm 0.0291)$ & $\beta_{13}$ & $\omega_{3}$ & $-5.952489( \pm 0.101)$ \\
\hline$\varepsilon_{17}$ & $e_{22}$ & $0.748031( \pm 0.0221)$ & $\beta_{14}$ & $\omega_{4}$ & $4.726133( \pm 0.0772)$ \\
\hline$\varepsilon_{18}$ & $e_{33}$ & $-0.051182( \pm 0.00167)$ & $\beta_{15}$ & $\psi$ & $-0.215487( \pm 0.00545)$ \\
\hline$\varepsilon_{19}$ & $e_{44}$ & $-0.002434( \pm 0.000324)$ & $\beta_{16}$ & $G^{\prime}$ & $0.000000( \pm 0.407)$ \\
\hline$\varepsilon_{20}$ & $e_{\mathrm{J}}$ & $0.002750( \pm 0.000081)$ & $\beta_{17}$ & $G$ & $-0.140855( \pm 0.00279)$ \\
\hline$\varepsilon_{21}$ & $c_{11}$ & $0.344275( \pm 0.0196)$ & $\beta_{18}$ & $\phi_{1}$ & $15^{\circ} 541000( \pm 0.411)$ \\
\hline$\varepsilon_{22}$ & $c_{22}$ & $-0.005970( \pm 0.000872)$ & $\beta_{19}$ & $\phi_{2}$ & $5.215000( \pm 0.469)$ \\
\hline$\varepsilon_{23}$ & $c_{33}$ & $0.041611( \pm 0.00199)$ & $\beta_{20}$ & $\phi_{3}$ & $-1.996000( \pm 0.757)$ \\
\hline$\varepsilon_{24}$ & $c_{44}$ & $-0.070074( \pm 0.000810)$ & $\beta_{21}$ & $\phi_{4}$ & $-7.968000( \pm 0.293)$ \\
\hline$\varepsilon_{25}$ & $I_{\mathrm{J}}$ & $0.005110( \pm 0.000079)$ & $\beta_{22}$ & $\Omega_{\mathrm{J}}$ & $0.045266( \pm 0.00664)$ \\
\hline
\end{tabular}

\subsection{Doppler data}

The Doppler observations discussed by Ostro et al. (1992) were employed to evaluate the ephemerides and explore the potential of Doppler data, but they were not included in analysis and the development of E5. The data are consistent with the observations which were analyzed, but they were not included in the analysis because of possible uncertainty in the radar scattering properties of the satellites similar to albedo effects which depend upon the planetocentric longitude. The 50 Doppler observations of the outer three Galilean satellites were made between 1987 and 1991.

The Doppler data were weighted using standard deviations of $19 \mathrm{~Hz}$ for Europa, $12 \mathrm{~Hz}$ for Ganymede and $10 \mathrm{~Hz}$ for Callisto for the Arecibo $13-\mathrm{cm} S$-band system data.

\section{Discussion}

The theory parameters which result from the analysis of these data are listed in Table 6 , which will produce coordinates in the B1950 frame when used with the galsat software. A future paper will document how they, and any other set of galsat parameters, can be transformed to the J2000 system in a manner such that the galsat software will directly produce J2000 coordinates. In Table 6, the uncertainties listed for the $\varepsilon$ and $\beta$ parameters are the formal errors obtained in the estimation process. By comparing the coordinates of ephemerides E3 with those of E5 and interpreting those differences to represent a 1- $\sigma$ error, we obtain a scale factor which should be applied for the formal uncertainties listed in the table. That scale factor ranges between 2.5 and 3 , so we recommend that the formal errors be multiplied by 3 . The derived values of the angular variables for E5 are given in Table 7. The series coefficients for satellite coordinates $\xi, v$ and $\zeta$ are summarized in Table 8 for the E5 ephemerides.

Representing the Jupiter-equatorial projection of the orbital radius by $\rho$, and the true and mean longitudes by $\nu$ and $\ell$, respectively, then the equatorial radial component $\xi=(\rho-a) / a$ consists of cosine terms $\xi(t)=$ $\Sigma K_{1} \cos \Theta_{1}(t)$, while the longitude component $v=\nu-\ell$ consists of sine terms $v(t)=\Sigma K_{2} \sin \Theta_{2}(t)$, and the latitude component $\zeta=\bar{z} / a$ consists of sine terms $\zeta(\tau)=$ 
Table 7. Derived variables for ephemeris E5

\begin{tabular}{|c|c|c|c|}
\hline$\overline{\text { Index }}$ & Variable & Value (deg) & Rate $($ deg/day $)$ \\
\hline 1 & $\ell_{1}$ & $106^{\circ} .077187$ & $203^{\circ} .48895579033$ \\
\hline 2 & $\ell_{2}$ & $175^{\circ} .731615$ & $101^{\circ} .37472473479$ \\
\hline 3 & $\ell_{3}$ & $120^{\circ} .558829$ & $50^{\circ} .31760920702$ \\
\hline 4 & $\ell_{4}$ & $84^{\circ} .444587$ & $21^{\circ} .57107117668$ \\
\hline 5 & $\phi_{\lambda}$ & 199.676608 & 0.17379190461 \\
\hline 6 & $\pi_{1}$ & $97^{\circ} .088086$ & 0.16138586144 \\
\hline 7 & $\pi_{2}$ & $154^{\circ} .866335$ & 0.04726306609 \\
\hline 8 & $\pi_{3}$ & $188^{\circ} .184037$ & 0.00712733949 \\
\hline 9 & $\pi_{4}$ & $335^{\circ} .286807$ & 0.00183999637 \\
\hline 10 & $\Pi_{\mathrm{J}}$ & $13^{\circ} .469942$ & 0 . \\
\hline 11 & $\omega_{1}$ & $312^{\circ} .334566$ & $-0^{\circ} .13279385940$ \\
\hline 12 & $\omega_{2}$ & $100^{\circ} .441116$ & $-0^{\circ} .03263063731$ \\
\hline 13 & $\omega_{3}$ & $119^{\circ} .194241$ & $-0^{\circ} .00717703155$ \\
\hline 14 & $\omega_{4}$ & $322^{\circ} .618633$ & $-0^{\circ} .00175933880$ \\
\hline 15 & $\psi$ & $316^{\circ} .518203$ & $-2^{\circ} .08362 \cdot 10^{-6}$ \\
\hline 16 & $G^{\prime}$ & $31^{\circ} .978528$ & 0.03345973390 \\
\hline 17 & $G$ & $30^{\circ} .237557$ & 0.08309257010 \\
\hline 18 & $\phi_{1}$ & $188^{\circ} .374346$ & 0. \\
\hline 19 & $\phi_{2}$ & $52^{\circ} .224824$ & 0 . \\
\hline 20 & $\phi_{3}$ & $257^{\circ} .184000$ & 0 . \\
\hline 21 & $\phi_{4}$ & $149^{\circ} .152605$ & 0 . \\
\hline 22 & $\Omega_{\mathrm{J}}$ & $99^{\circ} .998526$ & 0 . \\
\hline & $\begin{array}{l}a_{1} \\
a_{2} \\
a_{3} \\
a_{4}\end{array}$ & & $\begin{array}{r}2.819353 \cdot 10^{-3} \text { a.u. } \\
4.485883 \cdot 10^{-3} \text { a.u. } \\
7.155366 \cdot 10^{-3} \text { a.u. } \\
12.585464 \cdot 10^{-3} \text { a.u. }\end{array}$ \\
\hline
\end{tabular}

$\Sigma K_{3} \sin \Theta_{3}(\tau)$. As developed by Sampson (1921, pp. 229$230)$, the "time-completed" $\tau$ may be defined as

$$
\tau=t+v / n
$$

where $t$ is "ephemeris time" (TDB). One can employ the time-completed to compute the latitude quantity $s(t)=$ $\bar{z} / \rho$ from the shorter series for $\zeta(t)=\bar{z} / a$ via the relationship $s(t)=\zeta(t+v / n)$. It effectively amounts to calculating the latitude perturbations as a function of true longitude rather than as a function of mean longitude.

The Jupiter equatorial coordinates $\overline{\mathbf{r}}=(\bar{x}, \bar{y}, \bar{z})^{T}$ are computed from the orbital components $\xi, v, \zeta$ using the equations

$$
\begin{aligned}
& \bar{x}=a(1+\xi) \cos (\ell-\psi+v) \\
& \bar{y}=a(1+\xi) \sin (\ell-\psi+v) \\
& \bar{z}=a(1+\xi) s .
\end{aligned}
$$

The Earth-equatorial coordinates $\mathbf{r}=(x, y, z)^{T}$ are then computed from the Jupiter-equatorial coordinates via the rotation matrices

$$
\mathbf{r}=R_{1}(-\varepsilon) R_{3}(-\Omega) R_{1}(-J) R_{3}(-\psi+\Omega) R_{1}(-I) \overline{\mathbf{r}} .
$$

It is these Earth-equatorial coordinates $\mathbf{r}$ that are provided by the galsat software.

As described in Theory, the Earth-equatorial coordinates are constructed from the series for $\xi, v$ and $\zeta$ by the relationship

$$
\begin{aligned}
\xi(t) & =\Sigma K_{1} \cos \Theta_{1}(t) \\
v(t) & =\Sigma K_{2} \sin \Theta_{2}(t) \\
s(t)=\zeta(\tau) & =\Sigma K_{3} \sin \Theta_{3}(\tau)
\end{aligned}
$$

where the right-hand sides are the result of computing the series given in Table 8 . The third equation for $s(t)$ employs the time-completed $\tau=t+v / n$ to evaluate the series for $\zeta(\tau)$ and thus to obtain $s(t)$.

The adjustable parameters $\varepsilon$ and $\beta$ for ephemerides E5 in the B1950 frame are given in Table 6. The derived values of the angular variables for E5 are given in Table 7.

Acknowledgements. This paper represents the results of one phase of research conducted at the Jet Propulsion Laboratory, California Institute of Technology, under contract with the National Aeronautics and Space Administration. The CCD observations were made by $\mathrm{D}$. and A. Monet of the USNO Flagstaff Station and were processed into right-ascension and declination normal-point residuals on a fixed ephemeris by W.M. Owen Jr at JPL.

\section{References}

Aksnes K., 1974, Icarus 21, 100

Aksnes K., Franklin F., 1976, AJ 81, 464

Aksnes K., Franklin F., 1978, Icarus 34, 188

Aksnes K., 1984, Icarus 60, 180

Aksnes K., Franklin F., Millis R., et al., 1984, AJ 89, 28; AJ 89, 1081

Aksnes K., Franklin F., Magnusson P., 1986, AJ 92, 1436

Aksnes K., Franklin F., 1990, Icarus 84, 542

Aksnes K., 1993 (personal communication)

Arlot J.-E., 1978, A\&AS 34, 195

Arlot J.-E., 1982, A\&A 107, 305

Arlot J.-E., 1984, A\&A 138, 113

Arlot J.-E., 1990, A\&A 237, 259

Arlot J.-E., 1996, A\&A 314, 312

Bigourdan G., 1897, "Inventaire général et sommaire des manuscrits de la bibliothèque de l'observatoire Paris" Ann. Obs. Paris 21, F1-F60. [Delisle manuscripts are filed under the heading Manuscripts A-5-1 through A-5-8]

Bigourdan G., 1901, A.-G. Pingré: Annales Célestes du dixseptiéme siècle. Paris: Gauthier-Villars

Brouwer D., 1952, AJ 57, 126

Campbell J.K., Synnott S.P., 1985, AJ 90, 364

Davies M.E., Abalakin V.K., Bursa M., et al., 1986, Celest. Mech. 39, 103 
Descamps P., 1994, A\&A 291, 664

Franklin F., et al., "Galilean Satellite Observers", 1991, AJ 102,806

Franklin F., 1993 (personal communication)

Gaskell R.W., 1995 (personal communication)

Goguen J.D., Sinton W.M., Matson D.L., et al., 1988, Icarus 76,465

Goguen J.D., 1994 (personal communication)

Kaas A.A., Franklin F., Aksnes K., Lieske J.H., 1997, "Mutual phenomena of the Galilean satellites 1990-1991", AJ (in press)

Lieske J.H., 1977, A\&A 56, 333 (referred to as Theory)

Lieske J.H., Lederle T., Fricke W., Morando B., 1977, A\&A 58,1

Lieske J.H., 1979, A\&A 73, 28

Lieske J.H., 1980, A\&A 82, 340 [referred to as E2 ephemerides]

Lieske J.H., 1986a, A\&A 154, 61

Lieske J.H., 1986b, A\&AS 63, 143

Lieske J.H., 1987, A\&A 176, 146

Lieske J.H., 1994a, "Galilean Satellite Ephemerides E4" JPL Engineering Memorandum 314-545 (19 June 1994) (JPL internal publication)

Lieske J.H., 1994b, A\&A 281, 281

Lieske J.H., 1995, Bull. AAS 27, 1197

Lindegren L., 1977, A\&A 57, 55

Mallama A., 1992a, Icarus 95, 309

Mallama A., 1992b, Icarus 97, 298

Mallama A., 1993, J. Geophys. Res. 98, p. 18.873-18.876

Martin C.F., 1969, Ph.D. Diss., Yale Univ.

Monet A.K.B., Stone R.C., Monet D.G., et al., 1994, AJ 107, 2290

Monet D.G., Monet A.K.B., 1992, "Galilean satellite astrometry", U.S. Naval Observatory Flagstaff Station memorandum

Monet D.G., Dahn C.C., Vrba F.J., et al., 1992, AJ 103, 638
Morrison L.V., Ward C.G., 1975, MNRAS 173, 183

Morrison L.V., 1980 (personal communication)

Ostro S.J., Campbell D.B., Simpson R.A., et al., 1992, J. Geophys. Res. 97, p. 18.277

Owen W.M., 1993 (personal communication)

Owen W.M., 1995 (personal communication)

Pascu D., 1977, in: Planetary Satellites, Burns J.A. (ed.). University of Arizona Press, Tucson, p. 63

Pascu D., 1979, in: Natural and Artificial Satellite Motion Nacozy P.E., Ferraz-Mello S. (eds.). University of Texas, Austin, p. 17

Pascu D., 1993 (personal communication)

Pascu D., 1994, in: Galactic and Solar System Optical Astrometry, Morrison L.V., Gilmore G.F. (eds.). Cambridge University Press, p. 304

Pierce D.A., 1980, "Observations of Jupiter's Satellites", JPL Engineering Memorandum 900-672

Pickering E.C., 1907, Harvard Ann. 52, Part I, 1

Riedel J.E., 1994 (personal communication)

Sampson R.A., 1921, MRAS 63 (Sampson Theory)

Spencer J., 1993 (personal communication)

Spencer Jones H., 1939, MNRAS 99, 541

Standish E.M., 1982, A\&A 114, 297

Standish E.M., 1985, "JPL Planetary Ephemerides DE125", JPL IOM 314.6-591

Standish E.M., Folkner W.M., 1995, "JPL Ephemerides DE400 and DE140", JPL IOM 314.10-109

Stephenson F.R., Morrison L.V., 1984, Phil. Trans. R. Soc. London, Ser. A, 313, 47

Synnott S.P., Donegan A.J., Morabito L.A., 1982, "Position Observations of the Galilean Satellites from Voyager Data", Jet Propulsion Laboratory internal document

Vasundhara R., 1994, A\&A 281, 565

West R.M, 1992, in: Trans IAU XXIB, Bergeron J. (ed.). Kluwer, Dordrecht, p. 211 
Table 8. Series coefficients for E5

\begin{tabular}{|c|c|c|c|}
\hline Index & E5 & Argument & Ratio $n / n_{\text {sat }}$ \\
\hline \multicolumn{4}{|c|}{ XI-1: Series coefficients for $\xi_{1}=\left(\rho_{1}-a_{1}\right) / a_{1}$ (cosine) } \\
\hline 1 & 170 & $\ell_{1}-\ell_{2}$ & .50181707 \\
\hline 2 & 106 & $\ell_{1}-\ell_{3}$ & .75272560 \\
\hline 3 & -2 & $\ell_{1}-\pi_{1}$ & . 99920691 \\
\hline${ }_{5}^{4}$ & $\begin{array}{r}-2 \\
-387\end{array}$ & $\begin{array}{l}\ell_{1}-\pi_{2} \\
\ell_{1}-\pi_{3}\end{array}$ & .99976774 \\
\hline $\begin{array}{l}5 \\
6\end{array}$ & $\begin{array}{l}-387 \\
-214\end{array}$ & $\begin{array}{l}\ell_{1}-\pi_{3} \\
\ell_{1}-\pi_{4}\end{array}$ & 99996997 \\
\hline 7 & & $\begin{array}{l}\ell_{1}-\pi_{4} \\
\ell_{1}+\pi_{3}-2 \Pi_{J}-2 G\end{array}$ & . .999921835 \\
\hline 8 & -41339 & $2 \ell_{1}-2 \ell_{2}$ & 1.00363413 \\
\hline & & $2 \ell_{1}-2 \ell_{3}$ & \\
\hline 10 & & $4 \ell_{1}-4 \ell_{2}$ & 2.00726827 \\
\hline \multicolumn{4}{|c|}{ V-1: Series coefficients for $v_{1}=\nu_{1}-\ell_{1}$ (sine) } \\
\hline 1 & -26 & $-2 \Pi_{J}+2 \psi-2 G$ & -.00081670 \\
\hline & -553 & $-2 \Pi_{J}+2 \psi$ & \\
\hline 3 & $\begin{aligned}-240 \\
92\end{aligned}$ & $-2 \Pi_{J}+\omega_{3}+\psi-2 G$ & -.00085196 \\
\hline 4 & $\begin{array}{r}92 \\
-72\end{array}$ & $\begin{array}{l}-\omega_{2}+\psi \\
-\omega_{3}+\psi\end{array}$ & .00016035 \\
\hline 5 & $\begin{array}{l}-72 \\
-49\end{array}$ & $\begin{array}{l}-\omega_{3}+\psi \\
-\omega_{4}+\psi\end{array}$ & .00003526 \\
\hline${ }_{7}^{6}$ & $\begin{array}{l}-49 \\
-325\end{array}$ & $G$ & . \\
\hline & & $2 G$ & .00081668 \\
\hline & -33 & $5 G^{\prime}-2 G+\phi_{2}$ & .00000547 \\
\hline 10 & -27 & $\omega_{3}-\omega_{4}$ & -.00002662 \\
\hline 11 & 145 & $\omega_{2}-\omega_{3}$ & -.00012509 \\
\hline 12 & 30 & $\omega_{2}-\omega_{4}$ & -.00015171 \\
\hline 13 & -38 & $\pi_{4}-\Pi_{J}$ & .00000904 \\
\hline 14 & -6071 & $\pi_{3}-\pi_{4}$ & . .00002598 \\
\hline 15 & ${ }_{156}^{282}$ & $\pi_{2}-\pi_{3}$ & 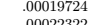 \\
\hline $\begin{array}{l}16 \\
17 \\
17\end{array}$ & $\begin{array}{c}156 \\
-38\end{array}$ & $\pi_{2}-\pi_{4}$ & . \\
\hline 18 & $\begin{array}{l}-38 \\
-25\end{array}$ & $\begin{array}{l}\pi_{1}-\pi_{3} \\
\pi_{1}-\pi_{4}\end{array}$ & - 00075807 \\
\hline 19 & -27 & $\pi_{1}+\pi_{4}-2 \Pi_{J}-2 G$ & -.00001454 \\
\hline 20 & -1176 & $\pi_{1}+\pi_{3}-2 \Pi_{J}-2 G$ & .00001144 \\
\hline 21 & 1288 & $\phi_{\lambda}$ & .00085406 \\
\hline & & $3 \hat{\ell}_{3}-7 \ell_{4}+4 \pi_{4}$ & -.00018335 \\
\hline 23 & -32 & $3 \ell_{3}-7 \ell_{4}+\pi_{3}+3 \pi_{4}$ & -.00015737 \\
\hline 24 & -1162 & $\ell_{1}-2 \ell_{2}+\pi_{4}$ & .00364318 \\
\hline 25 & -1887 & $\ell_{1}-2 \ell_{2}+\pi_{3}$ & . 00366916 \\
\hline 26 & -1244 & $\ell_{1}-2 \ell_{2}+\pi_{2}$ & . .00386640 \\
\hline${ }_{28}^{27}$ & 38 & $\ell_{1}-2 \ell_{2}+\pi_{1}$ & . .00442723 \\
\hline $\begin{array}{l}28 \\
29\end{array}$ & $\begin{array}{l}-617 \\
-270\end{array}$ & $\ell_{1}-\ell_{2}$ & . \\
\hline $\begin{array}{l}29 \\
30\end{array}$ & $\begin{array}{l}-2 \pi 0 \\
-26\end{array}$ & $\begin{array}{l}\ell_{1}-\ell_{3} \\
\ell_{1}-\ell_{4}\end{array}$ & - 893993900 \\
\hline 31 & $\begin{array}{r}-20 \\
4\end{array}$ & 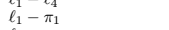 & $\begin{array}{l}.8999206901 \\
.999391\end{array}$ \\
\hline & 5 & $\ell_{1}-\pi_{2}$ & .99976774 \\
\hline 33 & 776 & & .99996497 \\
\hline 34 & 462 & $\ell_{1}-\pi_{4}$ & .99999096 \\
\hline 35 & 149 & $\ell_{1}+\pi_{3}-2 \Pi_{J}-2 G$ & .99921835 \\
\hline 36 & & & . .00707264 \\
\hline 37 & & $2 \ell_{1}-4 \ell_{2}+2 \omega_{2}$ & .00694756 \\
\hline 38 & 82483 & $2 \ell_{1}-2 \ell_{2}$ & 1.00363413 \\
\hline 39 & -35 & $2 \ell_{1}-2 \ell_{3}$ & 1.50545120 \\
\hline $\begin{array}{l}40 \\
41\end{array}$ & $\begin{array}{l}-3 \\
276\end{array}$ & $\begin{array}{l}3 \ell_{1}-4 \ell_{2}+\pi_{3} \\
4 \ell_{1}-4 \ell_{2}\end{array}$ & $\begin{array}{l}1.00730330 \\
2.0072627\end{array}$ \\
\hline & & $4 \ell_{1}-4 \ell_{2}$ & \\
\hline
\end{tabular}

\begin{tabular}{|c|c|c|c|}
\hline Index & E5 & Argument & Ratio $n / n_{\text {sat }}$ \\
\hline 22 & & & .00152167 \\
\hline & 111 & $\pi_{1}-\pi_{4}$ & .00157382 \\
\hline 24 & -354 & $\pi_{1}+\pi_{3}-2 \Pi_{J}-2 G$ & . .00002296 \\
\hline 25 & -3103 & $\phi_{\lambda}$ & .00171435 \\
\hline${ }_{27}^{26}$ & $\begin{array}{r}55 \\
-111\end{array}$ & $\begin{array}{l}2 \ell_{3}-2 \Pi_{J}-2 G \\
3 \ell_{3}-7 \ell_{1}+4 \pi_{1}\end{array}$ & .99106590 \\
\hline $\begin{array}{l}27 \\
28\end{array}$ & $\begin{array}{r}-111 \\
91\end{array}$ & $\begin{array}{l}3 \ell_{3}-7 \ell_{4}+4 \pi_{4} \\
3 \ell_{3}-7 \ell_{1}+\pi_{3}+3 \pi_{4}\end{array}$ & $\begin{array}{l}-.00036805 \\
-.00031589\end{array}$ \\
\hline $\begin{array}{l}28 \\
29\end{array}$ & $\begin{array}{r}91 \\
-25\end{array}$ & $\begin{array}{l}3_{3}-\ell_{4}+\pi_{3}+3 \pi_{4} \\
3 \ell_{3}-7 \ell_{4}+2 \pi_{3}+2 \pi_{4}\end{array}$ & $\begin{array}{l}-.00033589 \\
-.00026373\end{array}$ \\
\hline 30 & -1994 & $\ell_{2}-\ell_{3}+2 \pi_{3}+2 \pi_{4}$ & $\begin{array}{r}-.000363733 \\
.5034739\end{array}$ \\
\hline 31 & -137 & $\ell_{2}-\ell_{4}^{3}$ & .78721450 \\
\hline 32 & 1 & $\ell_{2}-\pi_{1}$ & .99840803 \\
\hline 33 & 2886 & $\begin{array}{l}\ell_{2}-\pi_{2} \\
\ell_{\ell}-\pi_{3}\end{array}$ & .99953378 \\
\hline 34 & 6250 & $\begin{array}{l}\ell_{2}-\pi_{3} \\
\ell_{2}-\pi_{4}\end{array}$ & .99992969 \\
\hline $\begin{array}{l}35 \\
36\end{array}$ & 3463 30 & $\begin{array}{l}\ell_{2}-\pi_{4} \\
\ell_{2}-\Pi_{J}-G\end{array}$ & .99998185 \\
\hline & -18 & $\begin{array}{l}\ell_{2}-11 J-G \\
2 \ell_{2}-3 \ell_{3}+\pi_{4}\end{array}$ & $\begin{array}{l}999180034 \\
5909632\end{array}$ \\
\hline 38 & $\begin{array}{l}-18 \\
-39\end{array}$ & $2 \ell_{2}-3 \ell_{3}+\pi_{3}$ & $\begin{array}{l}510960322 \\
.51101248\end{array}$ \\
\hline 39 & 98 & $2 \ell_{2}-2 \ell_{4}$ & 1.57442901 \\
\hline 40 & -164 & $2 \ell_{2}-2 \omega_{2}$ & 2.00064376 \\
\hline 41 & -18 & $2 \ell_{2}-\omega_{2}-\omega_{3}$ & 2.00039268 \\
\hline 42 & 72 & $5 \ell_{2}-5 \ell_{3}$ & 2.51823695 \\
\hline $\begin{array}{l}43 \\
44\end{array}$ & $\begin{array}{r}30 \\
4180\end{array}$ & $\begin{array}{l}\ell_{1}-2 \ell_{2}-\pi_{3}+2 \Pi_{J}+2 G \\
\ell_{1}-2 \ell_{2}+\pi\end{array}$ & $\begin{array}{l}.00886379 \\
0073293\end{array}$ \\
\hline & $\begin{array}{l}4180 \\
7428\end{array}$ & $\begin{array}{l}\ell_{1}-2 \ell_{2}+\pi_{4} \\
\ell_{1}-2 \ell_{2}+\pi_{3}\end{array}$ & $\begin{array}{l}.007319293 \\
.0073609\end{array}$ \\
\hline 46 & -2329 & $\ell_{1}-2 \ell_{2}+\pi_{2}$ & . .00776100 \\
\hline 47 & -19 & $\ell_{1}-2 \ell_{2}+\pi_{1}$ & .00888675 \\
\hline 48 & -185835 & $\ell_{1}-\ell_{2}$ & 1.00729478 \\
\hline 49 & -110 & $\ell_{1}-2 \ell_{3}+\pi_{4}$ & 1.01460771 \\
\hline 50 & -200 & $\ell_{1}-2 \ell_{3}+\pi_{3}$ & $\begin{array}{l}1.01465987 \\
10150578\end{array}$ \\
\hline $\begin{array}{l}51 \\
52\end{array}$ & $\begin{array}{r}39 \\
-16\end{array}$ & $\begin{array}{l}\ell_{1}-2 \ell_{3}+\pi_{2} \\
\ell\end{array}$ & $\begin{array}{l}1.015015578 \\
1.01618153\end{array}$ \\
\hline 53 & $\begin{array}{l}-10 \\
-803\end{array}$ & $\begin{array}{l}\ell_{1}-2 \ell_{3}+\pi_{1} \\
\ell_{1}-\ell_{3}\end{array}$ & $\begin{array}{l}1.016169153 \\
1.5104217\end{array}$ \\
\hline 54 & -19 & $\begin{array}{l}1 \\
\ell_{1}-\pi_{2}\end{array}$ & $\begin{array}{l}1.01094211 \\
2.00682856\end{array}$ \\
\hline 55 & -75 & $\ell_{1}-\pi_{3}$ & 2.00722447 \\
\hline 56 & -31 & $\ell_{1}-\pi_{4}$ & 2.00727663 \\
\hline 57 & -9 & $2 \ell_{1}-4 \ell_{2}+\omega_{3}+\psi$ & .01451874 \\
\hline 58 & 4 & $2 \ell_{1}-4 \ell_{2}+2 \omega_{3}$ & . 01444797 \\
\hline $\begin{array}{l}59 \\
60\end{array}$ & -14 & $2 \ell_{1}-4 \ell_{2}+\omega_{2}+\omega_{3}$ & . 01419658 \\
\hline 61 & $\begin{array}{c}111 \\
-11\end{array}$ & $\begin{array}{l}22 \ell_{1}-4 \ell_{2}+2 \omega_{2} \\
2 \ell_{1}-4 \ell_{2}+2 \Pi_{I}+2 G\end{array}$ & . 01622888 \\
\hline $\begin{array}{l}01 \\
62\end{array}$ & $\begin{array}{l}-11 \\
-9\end{array}$ & $\begin{array}{l}2 \ell_{1}-4 \ell_{2}+21 I_{J}+2 G \\
2 \ell_{1}-4 \ell_{2}+\pi_{3}+\pi_{4}\end{array}$ & $\begin{array}{l}.014622888 \\
.0146702\end{array}$ \\
\hline 63 & -8 & $2 \ell_{1}-4 \ell_{2}+2 \pi_{3}$ & .01473017 \\
\hline 64 & 915 & $2 \ell_{1}-2 \ell_{2}$ & 2.01458956 \\
\hline 65 & 96 & $2 \ell_{1}-2 \ell_{3}^{2}$ & 3.02188434 \\
\hline \multirow[t]{2}{*}{66} & -18 & $4 \ell_{1}-4 \ell_{2}$ & 4.02917912 \\
\hline & \multicolumn{2}{|c|}{ AT-2: Series coefficients for $\zeta_{2}=z_{2} / a_{2}$ (sine) } & \\
\hline 1 & 17 & $\ell_{2}-2 \Pi_{J}+\psi-3 G$ & .99754101 \\
\hline${ }_{3}^{2}$ & 143 & $\ell_{2}-2 \Pi_{J}+\psi-2 G$ & .99836066 \\
\hline $\begin{array}{l}3 \\
4\end{array}$ & 81004 & $\begin{array}{l}l_{2}-\omega_{1} \\
\ell_{2}-\omega_{2}\end{array}$ & $\begin{array}{l}1.00130993 \\
1.00032188\end{array}$ \\
\hline $\begin{array}{l}4 \\
5\end{array}$ & $\begin{array}{r}81004 \\
4512\end{array}$ & $\ell_{2}-6$ & $\begin{array}{l}1.00032188 \\
1.00007080\end{array}$ \\
\hline 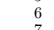 & 1160 & $\ell_{2}^{2}-\omega_{4}^{3}$ & 1.00001735 \\
\hline 7 & & $\ell_{2}-\psi-G$ & .99918036 \\
\hline 8 & -3284 & $\ell_{2}-\psi$ & 1.00000002 \\
\hline${ }_{10}^{9}$ & $\begin{array}{r}35 \\
-28 \\
\end{array}$ & $\begin{array}{l}\ell_{2}-\psi+G \\
\ell_{-}-2 \ell_{2}+\omega_{3}\end{array}$ & $\begin{array}{l}1.00081968 \\
1.0145876\end{array}$ \\
\hline${ }_{11}^{10}$ & $\frac{-28}{272}$ & $\begin{array}{l}\ell_{1}-2 \ell_{3}+\omega_{3} \\
\ell_{1}-2 \ell_{3}+\omega_{2}\end{array}$ & $\begin{array}{l}1.01451876 \\
1.01426768\end{array}$ \\
\hline
\end{tabular}

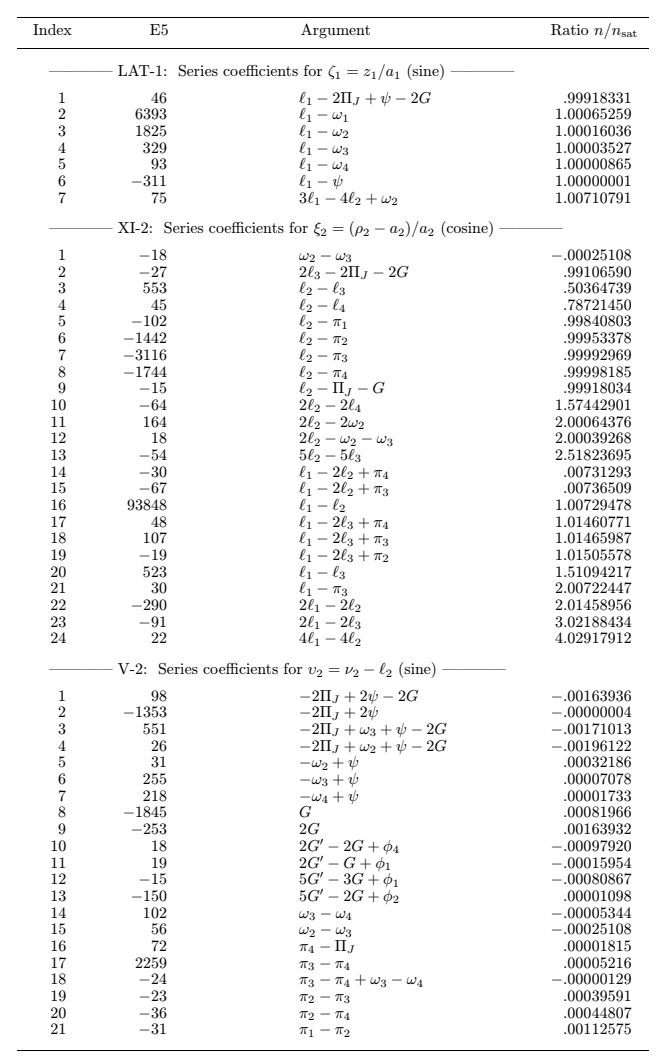

\begin{tabular}{|c|c|c|c|}
\hline Index & E5 & Argument & Ratio $n / n_{\text {sat }}$ \\
\hline \multicolumn{4}{|c|}{ XI-3: Series coefficients for $\xi_{3}=\left(\rho_{3}-a_{3}\right) / a_{3}$ (cosine) } \\
\hline \multirow{15}{*}{$\begin{array}{l}2 \\
3 \\
4 \\
5 \\
6 \\
7 \\
8 \\
9 \\
10 \\
11 \\
12 \\
13 \\
14 \\
16 \\
17 \\
18 \\
19 \\
0\end{array}$} & 24 & $-\omega_{3}+\psi$ & . 00014259 \\
\hline & $\begin{array}{l}-99 \\
10 \\
10\end{array}$ & $\begin{array}{llll}\omega_{3}-\omega_{4} \\
\pi_{3}-\pi_{4}\end{array}$ & $\begin{array}{c}-.00010767 \\
.0010508 \\
\end{array}$ \\
\hline & ${ }_{18}^{294}$ & $\begin{array}{l}\ell_{3-}^{3}- \\
\ell_{3}-\end{array}$ & $\begin{array}{l}.57330175 \\
.9990671\end{array}$ \\
\hline & $\begin{array}{l}-14388 \\
-7919\end{array}$ & $\begin{array}{l}\ell_{3}^{3}-\pi_{3}^{2} \\
\ell_{3}-\pi_{4}\end{array}$ & 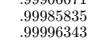 \\
\hline & ${ }_{-20}^{-23}$ & 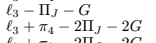 & 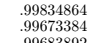 \\
\hline & $\begin{array}{r}-51 \\
39 \\
-761\end{array}$ & $\begin{array}{l}l_{3}+\pi_{3}-211 J-2 G \\
2 \ell_{3}-3 \ell_{4}+\pi_{4}\end{array}$ & 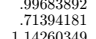 \\
\hline & $\begin{array}{r}-1761 \\
-11 \\
-10\end{array}$ & 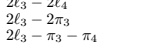 & $\begin{array}{l}1.1 .999717671 \\
1.999879 \\
1.979\end{array}$ \\
\hline & $\begin{array}{l}-27 \\
-24\end{array}$ & $\begin{array}{l}2 \ell_{3}-2 \Pi_{J}-2 G \\
2 \ell_{3}-2 \omega_{3}-2 G\end{array}$ & $\begin{array}{l}1.999821179 \\
1.99602828 \\
2.20028527\end{array}$ \\
\hline & $\begin{array}{c}99 \\
-24\end{array}$ & $\begin{array}{ll}2 & 2 l_{3}-\omega_{3}-\omega_{4} \\
2 l_{3}-\omega_{3}-\psi & -\psi\end{array}$ & 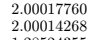 \\
\hline & $\begin{array}{c}-166 \\
-156\end{array}$ & $\begin{array}{l}3 \ell_{3}-4 \ell_{4}+\pi_{4} \\
3 \ell_{3}-3 \ell_{4}\end{array}$ & $\begin{array}{l}1.285242335 \\
1.7139524 \\
-\end{array}$ \\
\hline & $\begin{array}{l}-42 \\
-11\end{array}$ & $\begin{array}{l}4 \ell_{3}-4 \ell_{4} \\
5 \ell_{3}-5 \ell_{4}\end{array}$ & 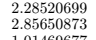 \\
\hline & 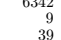 & $\begin{array}{l}\ell_{2}-l_{3} \\
\ell_{2}-\pi_{3} \\
2 \ell_{2}-3 \ell_{2}\end{array}$ & $\begin{array}{l}1.01469677 \\
2.0145512 \\
202512\end{array}$ \\
\hline & $\begin{array}{c}39 \\
70 \\
70\end{array}$ & 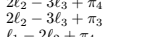 & 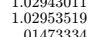 \\
\hline & $\begin{array}{c}10 \\
20 \\
152\end{array}$ & $\begin{array}{ll}\ell_{1}-2 \ell_{2}+\pi_{4} \\
\ell_{1}-2 \ell_{2}+\pi_{3}\end{array}$ & $\begin{array}{l}.014733384 \\
.0148342\end{array}$ \\
\hline & 156 & $\begin{array}{ll}\ell_{1}-2_{2} \\
\ell_{1}-\ell_{3}\end{array}$ & $\begin{array}{l}2.2029393354 \\
3.04409031\end{array}$ \\
\hline \multirow{14}{*}{ 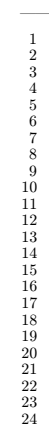 } & $\mathrm{V}-3: \mathrm{Se}$ & or $v_{3}=\nu_{3}-\ell_{3}$ (sine) & \\
\hline & ${ }_{28}^{10}$ & $-\pi_{3}+\pi_{4}-\omega_{3}+\psi$ & \\
\hline & -1770 & $\begin{array}{l}-211 J+2 \psi-2 G \\
-2 \Pi J+2 \psi\end{array}$ & . 0000000008 \\
\hline & ${ }_{14}^{-48}$ & $\begin{array}{l}-211 J+\omega_{3}+\psi-2 G \\
-\omega_{2}+\psi\end{array}$ & 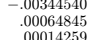 \\
\hline & 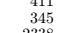 & $\begin{array}{l}-\omega_{3}+\psi \\
-\omega_{4}+\psi\end{array}$ & .00003492 \\
\hline & $\begin{aligned}-2338 \\
-66\end{aligned}$ & & 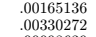 \\
\hline & $\begin{array}{l}10 \\
22 \\
20\end{array}$ & 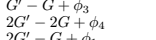 & . 0009967278 \\
\hline & $\begin{array}{c}26 \\
11 \\
9 \\
9\end{array}$ & 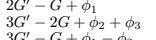 & 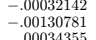 \\
\hline & $\begin{array}{r}-19 \\
-298 \\
\end{array}$ & $\begin{array}{l}5 G^{\prime}-3 G+\phi_{1}-\phi_{2} \\
5 G^{\prime}-2 G+\phi_{2}\end{array}$ & . 000629233 \\
\hline & 159 & $\omega_{3}-\omega_{4}+\phi_{2}$ & .00010767 \\
\hline & ${ }_{121}^{21}$ & $\begin{array}{l}\omega_{2}-\omega_{3} \\
\pi_{4}-\Pi_{J}\end{array}$ & .0000005656 \\
\hline & $\begin{array}{l}6604 \\
-65\end{array}$ & $\begin{array}{l}\pi_{3}-\pi_{4} \\
\pi_{3}-\pi_{4}+\omega_{3}-\omega_{4}\end{array}$ & . 000000259 \\
\hline & $\begin{array}{l}-88 \\
-72\end{array}$ & $\begin{array}{l}\pi_{2}-\pi_{3} \\
\pi_{2}-\pi_{4}\end{array}$ & .00079765 0.00273 \\
\hline & & & \\
\hline
\end{tabular}


Table 8. continued

\begin{tabular}{|c|c|c|c|}
\hline Index & E5 & Argument & Ratio $n / n_{\text {sat }}$ \\
\hline $\begin{array}{l}25 \\
26 \\
27 \\
28 \\
29 \\
30 \\
31 \\
32 \\
33 \\
34 \\
35 \\
36 \\
37 \\
38 \\
39 \\
40 \\
41 \\
42 \\
43 \\
44 \\
45 \\
46 \\
47 \\
48 \\
49 \\
50 \\
51 \\
52 \\
53 \\
54 \\
55 \\
56 \\
57 \\
58 \\
59 \\
60 \\
61 \\
62 \\
63 \\
64 \\
65 \\
66 \\
67 \\
68 \\
69 \\
70 \\
71 \\
72 \\
73 \\
74 \\
75\end{array}$ & $\begin{array}{r}-9 \\
16 \\
125 \\
307 \\
-10 \\
-100 \\
83 \\
-944 \\
-347 \\
28770 \\
1589 \\
7 \\
46 \\
41 \\
51 \\
11 \\
97 \\
1 \\
-101 \\
13 \\
3222 \\
29 \\
25 \\
37 \\
-24 \\
-94 \\
-174 \\
140 \\
-55 \\
27 \\
227 \\
53 \\
13 \\
42 \\
-12055 \\
-24 \\
-70 \\
-79 \\
-661 \\
-1328 \\
1082 \\
90 \\
190 \\
218 \\
2 \\
-4 \\
3 \\
2 \\
2 \\
-13\end{array}$ & 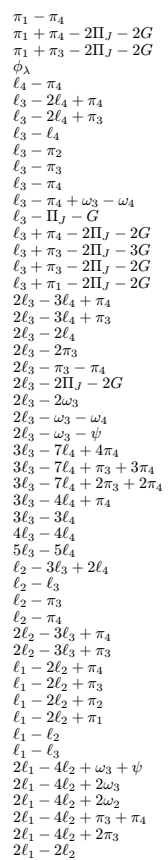 & 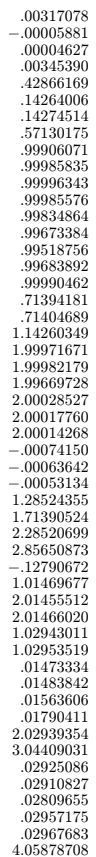 \\
\hline $\begin{array}{l}4 \\
5\end{array}$ & $\begin{array}{r}- \text { LAT-3: } \\
37 \\
321 \\
-15 \\
-45 \\
-2797\end{array}$ & $\begin{array}{l}\text { ts for } \zeta_{3}=z_{3} / a_{3}(\text { sine) } \\
\ell_{3}-2 \Pi_{J}+\psi-3 G \\
\ell_{3}-2 \Pi_{J}+\psi-2 G \\
\beta_{3}-2 \Pi_{J}+\psi-G \\
l_{3}-2 \Pi_{J}-\psi \\
\ell_{3}-\omega_{2}\end{array}$ & $\begin{array}{r}.995045877 \\
.99669724 \\
.99834860 \\
.99999996 \\
1.00064849\end{array}$ \\
\hline
\end{tabular}

\begin{tabular}{|c|c|c|c|}
\hline Index & E5 & Argument & Ratio $n / n_{\text {sat }}$ \\
\hline $\begin{array}{l}6 \\
7 \\
8 \\
9 \\
10 \\
11 \\
12 \\
13\end{array}$ & $\begin{array}{r}32402 \\
6847 \\
-45 \\
-16911 \\
51 \\
10 \\
-21 \\
30\end{array}$ & $\begin{array}{l}\ell_{3}-\omega_{3} \\
\ell_{3}-\omega_{4} \\
\ell_{3}-\psi-G \\
\ell_{3}-\psi \\
\ell_{3}-\psi+G \\
2 \ell_{2}-3 \ell_{3}+\psi \\
2 \ell_{2}-33_{3}+\omega_{3} \\
2 \ell_{2}-3 \ell_{3}+\omega_{2}\end{array}$ & $\begin{array}{l}1.00014263 \\
1.00003496 \\
1.99834868 \\
1.00000004 \\
1.00165140 \\
1.02939350 \\
1.092925091 \\
1.02874505\end{array}$ \\
\hline $\begin{array}{l}1 \\
2 \\
3 \\
4 \\
5 \\
6 \\
7 \\
8 \\
9 \\
10 \\
11 \\
12 \\
13 \\
14 \\
15 \\
16 \\
17 \\
18 \\
19 \\
20 \\
21 \\
22 \\
23 \\
24 \\
25 \\
26 \\
27 \\
28 \\
29 \\
30 \\
31 \\
32 \\
33 \\
34 \\
35 \\
36 \\
37 \\
38 \\
39 \\
40 \\
41 \\
42 \\
42\end{array}$ & $\begin{array}{r}-X I-4: \text { Se } \\
-19 \\
167 \\
11 \\
12 \\
-13 \\
1621 \\
-24 \\
-717 \\
-73546 \\
15 \\
30 \\
-5 \\
-89 \\
182 \\
-6 \\
-62 \\
-643 \\
27 \\
6 \\
6 \\
-9 \\
14 \\
13 \\
-271 \\
-25 \\
-155 \\
12 \\
48 \\
48 \\
-167 \\
142 \\
-22 \\
20 \\
974 \\
24 \\
117 \\
4 \\
42 \\
14 \\
5 \\
92 \\
92 \\
105\end{array}$ & 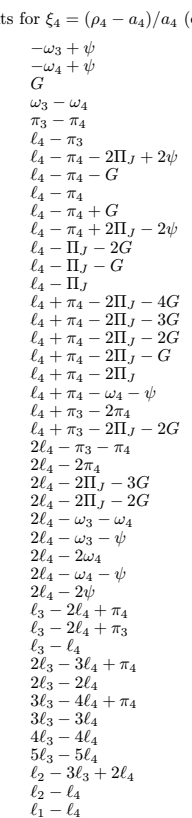 & 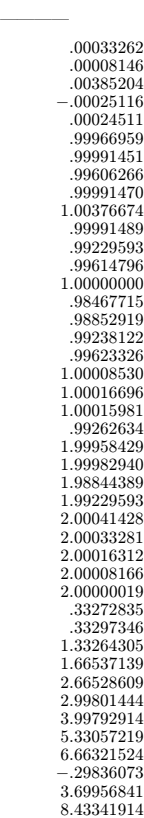 \\
\hline
\end{tabular}

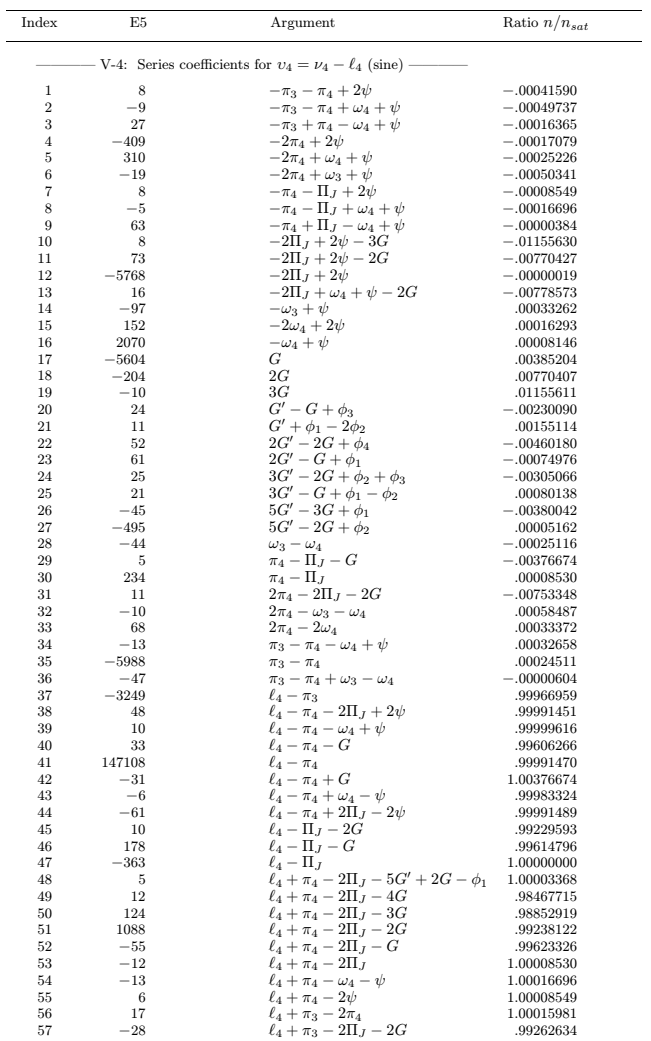

\begin{tabular}{|c|c|c|c|}
\hline Index & E5 & Argument & Ratio $n / n_{\text {sat }}$ \\
\hline $\begin{array}{l}58 \\
59 \\
60 \\
61 \\
62 \\
63 \\
64 \\
65 \\
66 \\
67 \\
68 \\
69 \\
69 \\
70 \\
71 \\
72 \\
73 \\
74 \\
75 \\
76 \\
77 \\
78 \\
79 \\
80 \\
81 \\
82 \\
83 \\
84 \\
85 \\
86\end{array}$ & $\begin{array}{r}-33 \\
676 \\
36 \\
218 \\
-5 \\
12 \\
-19 \\
-48 \\
-167 \\
-142 \\
148 \\
-94 \\
-390 \\
9 \\
-37 \\
6 \\
-195 \\
6 \\
187 \\
-149 \\
51 \\
-10 \\
6 \\
-8 \\
-41 \\
-13 \\
-44 \\
89 \\
106\end{array}$ & $\begin{array}{l}2 \ell_{4}-\pi_{3}-\pi_{4} \\
2 \ell_{4}-2 \pi_{4} \\
2 \ell_{4}-2 \Pi_{J}-3 G \\
2 \ell_{4}-2 \Pi_{J}-2 G \\
2 \ell_{4}-2 \Pi_{J}-G \\
2 \ell_{4}-\omega_{3}-\omega_{4} \\
2 \ell_{4}-\omega_{3}-\psi \\
2 \ell_{4}-2 \omega_{4} \\
2 \ell_{4}-\omega_{4}-\psi \\
\ell_{4}-2 \psi \\
\ell_{3}-2 \ell_{4}+\pi_{4} \\
\ell_{3}-2 \ell_{4}+\pi_{3} \\
2 \ell_{3}-4 \ell_{3}+2 \pi_{4} \\
2 \ell_{3}-3 \ell_{4}+\pi_{4} \\
2 \ell_{3}-3 \ell_{3}+\pi_{3} \\
3 \ell_{3}-2 \ell_{4} \\
3 \ell_{3}-7 \ell_{4}+2 \pi_{4}+\omega_{4}+\psi \\
3 \ell_{3}-7 \ell_{4}+4 \pi_{4} \\
3 \ell_{3}-7 \ell_{4}+2 \pi_{3}+3 \pi_{4} \\
3 \ell_{3}-7 \ell_{4}+3 \pi_{3}+\pi_{4} \\
3 \ell_{3}-6 \ell_{4}+3 \pi_{4} \\
3 \ell_{3}-444_{4}+\pi_{4} \\
4 \ell_{3}-4 \ell_{4} \\
\ell_{2}-3 \ell_{4}+2 \ell_{4} \\
\ell_{2}-\ell_{4} \\
\ell_{1}-\ell_{4}\end{array}$ & 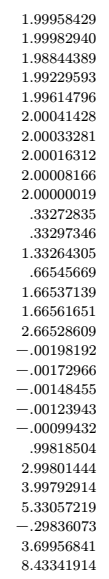 \\
\hline $\begin{array}{l}1 \\
2 \\
3 \\
4 \\
5 \\
6 \\
7 \\
8 \\
9 \\
10 \\
11 \\
12 \\
13 \\
14 \\
15 \\
16 \\
17 \\
18\end{array}$ & $\begin{array}{r}\text { AT-4: Se } \\
8 \\
8 \\
88 \\
773 \\
-38 \\
5 \\
9 \\
-17 \\
-5112 \\
-7 \\
44134 \\
7 \\
-102 \\
-76599 \\
104 \\
-10 \\
-11 \\
-11\end{array}$ & $\begin{array}{l}\text { or } \zeta_{4}=z_{4} / a_{4}(\text { sine }) \\
\ell_{4}-2 \Pi_{J}-\omega_{4}+2 \psi \\
\ell_{4}-2 \Pi_{J}+\psi-4 G \\
\ell_{4}-2 \Pi_{J}+\psi-3 G \\
\ell_{4}-2 \Pi_{J}+\psi-2 G \\
\ell_{4}-2 \Pi_{J}+\psi-G \\
\ell_{4}-2 \Pi_{J}+\psi \\
\ell_{4}-\omega_{1} \\
\ell_{4}-\omega_{2} \\
\ell_{4}-\omega_{3} \\
\ell_{4}-\omega_{4}-G \\
\ell_{4}-\omega_{4} \\
\ell_{4}-\omega_{4}+G \\
\ell_{4}-\psi-G \\
\ell_{4}-\psi+\psi \\
\ell_{4}-\psi+\psi \\
\ell_{3}-2 \ell_{4}+\psi \\
\ell_{3}-2 \ell_{4}+\omega_{4}^{\prime}-2 G+\phi_{2}\end{array}$ & 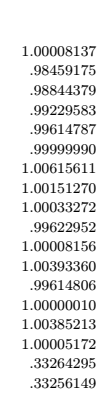 \\
\hline
\end{tabular}

